\title{
State-Space Approach to Nonlinear Predictive Generalized Minimum Variance Control
}

\author{
Industrial Control Centre \\ University of Strathclyde \\ Graham Hills Building \\ 50 George Street \\ GLASGOW GI IQE \\ United Kingdom
}

Mike J Grimble and Pawel Majecki

Telephone $N^{0}:+44(0) 1415524400$ Extensions: 2378/2880

Direct Line $\mathrm{N}^{\circ}:+44(0) 1415482378$

Facsimile $N^{\circ}: \quad+44(0) 1415484203$

Electronic Mail: m.grimble@eee.strath.ac.uk

http://www.icc.strath.ac.uk 


\begin{abstract}
A Nonlinear Predictive Generalized Minimum Variance (NPGMV) control al gorithm is introduced for the control of nonlinear discrete-time multivariable systems. The plant model is represented by the combination of a very general nonlinear operator and also a linear subsystem which can be open-loop unstable and is represented in state-space model form. The multi-step predictive control cost index to be minimised involves both weighted error and control signal costing terms. The solution for the control law is derived in the time-domain using a general operator representation of the process. The controller includes an internal model of the nonlinear process but because of the assumed structure of the system the state observer is only required to be linear. In the asymptotic case, where the plant is linear, the controller reduces to a state-space version of the well known GPC controller.
\end{abstract}

Keywords: State-space, predictive, nonlinear, optimal, minimum variance, transport delay.

Acknowledgements: We are grateful for the support of the EPSRC on the Platform Grant Project $N^{o}$ EP/C526422/1 and for the use of the ship model from the Marine Systems Simulator developed by the Norwegian University of Science and Technology. 


\section{Introduction}

The aim is to design a relatively simple controller for nonlinear systems that has some of the advantages of the popular Generalised Predictive Control (GPC) algorithms. The model based predictive control $(M B P C)$ approach based on linear theory has been applied very successfully in the process industries, where it has repeatedly improved the profitability and competitiveness of a production plant. It has been used to improve performance in difficult systems which contain long dead times, time-varying system parameters and multivariable interactions. Predictive algorithms were initially applied on relatively slow processes (such as thermal processes) for the chemical, petrochemical, food and cement industries but are now applied on faster systems, such as servo systems, hydraulic systems and gas turbine applications. Dynamic Matrix Control (DMC), due to Cutler and Ramaker [1] and Generalized Predictive Control (GPC), due to Clarke et. al. ([2], [3]) are popular. Richalet ([4], [5]) developed some of the first predictive controllers and has applied the technique successfully in a wide range of applications. The relationship between $L Q$ optimal and predictive control was explored in Bitmead et al [6]. A state-space version of a GPC controller was obtained in [7].

The solution presented here builds upon previous results on Generalised Minimum Variance $(G M V)$ control. A Nonlinear Generalized Minimum Variance (NGMV) controller was derived recently for nonlinear model based multivariable systems by Grimble ([8], [9]) and Grimble and Majecki [10]. The extension over the basic $N G M V$ control law involves an extension of the $N G M V$ cost-index to include future tracking error and control costing terms in a GPC type of problem. When the system is linear the results revert to those for a GPC controller which is a valuable solution for many applications. An advantage of the proposed predictive control approach is that the plant model can be in a general nonlinear operator form, which might involve 
hard nonlinearities, a state-dependent state-space model, transfer operators or even nonlinear function look up tables.

The possible advantages relative to other nonlinear predictive control approaches can be listed as:

- The general approach is close in spirit to fixed model based control so avoids problems with on-line linearization and behaviour should be easier to predict.

- If the system is close to being linear the system will behave like a linear GPC control design which is of course similar to DMC and many other well used and accepted techniques.

- No advanced concepts are needed to derive the solution presented here and this can be valuable in gaining acceptance from busy engineers in industry.

The road map for this paper is as follows. The nonlinear plant and linear state-space disturbance models are described in $\S 2$. It is shown in $\S 3$ that the solution of the linear multi-step predictive $(G P C)$ control problem can be found from the solution of an equivalent minimum variance control problem. The cost function and the solution of the NPGMV nonlinear optimal control problem are described in $\$ 4$ together with the main theorem. The stability and design issues are considered in $\$ 5$. An illustrative design example is presented in $\$ 6$. Finally conclusions that may be drawn are summarised in $\S 7$.

\section{System Models}

The plant model relating input and output can be grossly nonlinear, dynamic and may have a very general form, however, the disturbance signal is assumed to have a linear time-invariant model representation. The system in Fig. 1 includes the nonlinear plant model together with the linear reference, measurement noise and disturbance signals. 


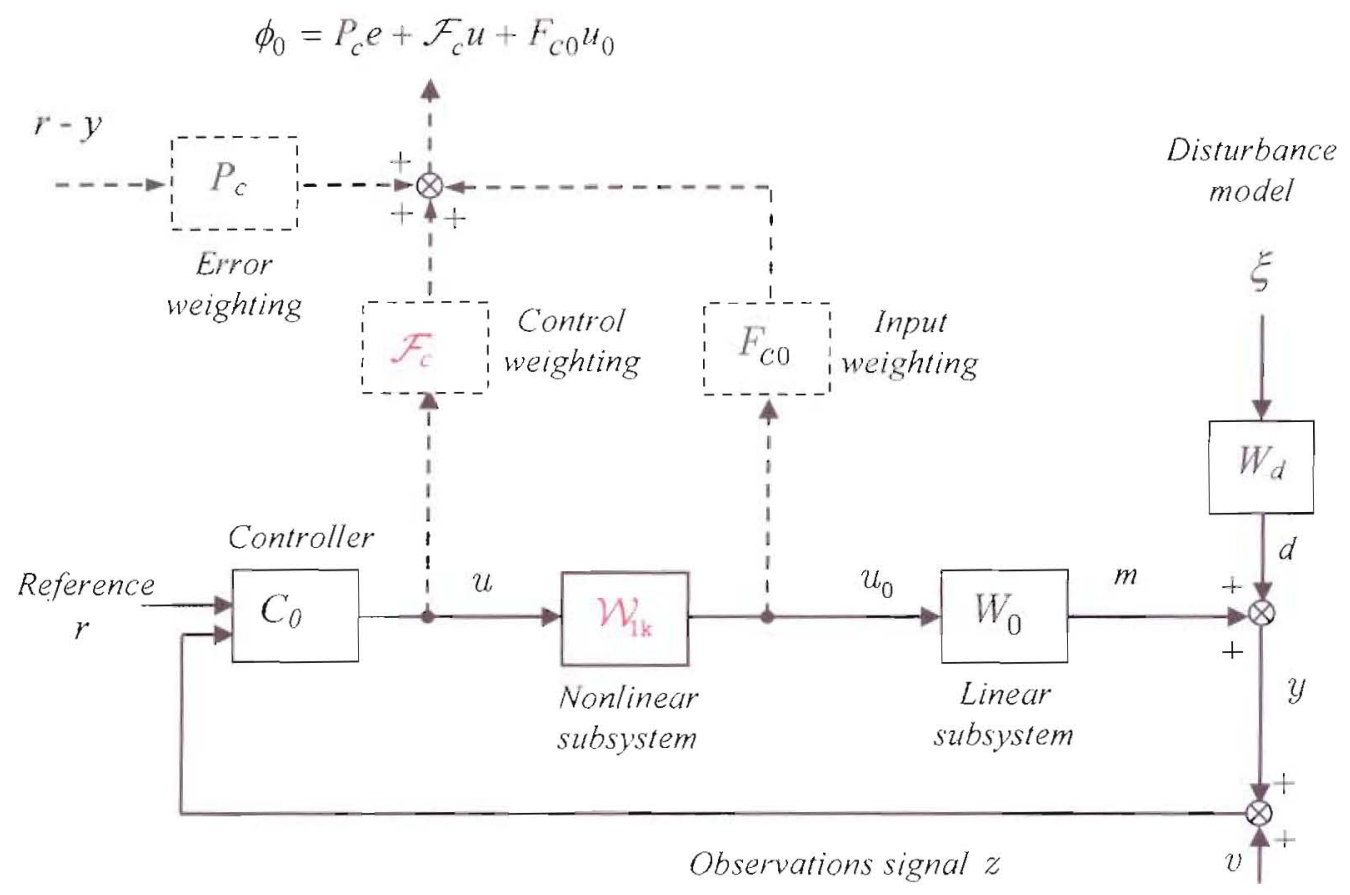

Fig. 1: Two Degrees of Freedom Feedback Control System for Nonlinear Plant

The signals $v(t)$ and $\xi(t)$ are vector zero-mean, independent, Gaussian white noise signals. The white measurement noise signal $\{v(t)\}$ is assumed to have a constant covariance matrix $R_{f}=R_{f}^{T} \geq 0$ and there is no loss of generality in assuming that the zero-mean white noise source $\{\xi(t)\}$ has an identity covariance matrix. It will be shown that there is no requirement to specify the distribution of the noise source, since the special structure of the system leads to a prediction equation, which is only dependent upon the linear stochastic disturbance model. The plant may have a very general nonlinear operator form.

\section{Nonlinear plant model: $\quad\left(\mathcal{W}_{1} u\right)(t)=z^{-k}\left(\mathcal{W}_{\mathrm{Ik}} u\right)(t)$}

where $z^{-k} I$ denotes a diagonal matrix of the common delay elements in the output signal paths. The output of the non-linear subsystem $\mathcal{W}_{k k}$ will be denoted as $u_{0}(t)=\left(\mathcal{W}_{1 \mathrm{k}} u\right)(t)$. For simplicity the nonlinear subsystem: $\mathcal{W}_{1}$ is assumed to be finite gain stable but the linear subsystem, denoted 
$W_{0}=z^{-k} W_{0 \mathrm{k}}$, is introduced in more detail below and can contain any unstable modes. If there is no linear sub-system component then $W_{0 k}=I$. The generalisation to different delays in different signal paths complicates the solution but is straightforward [9]. The weighted output equation can include any stable dynamic cost-function weighting $y_{p}(t)=P_{C}\left(z^{-1}\right) y(t)$.

Linear State-Space Subsystem Models

The first of the sub-systems to be defined is associated with the linear disturbance model and any linear sub-system $W_{0}$ in the plant model. Consider first the linear subsystems shown in Fig. 2. The linear sub-system model in Fig. 2 may be assumed to be stabilizable and detectable and to be represented in the state-space equation form:

$$
\begin{gathered}
x(t+1)=A x(t)+B u_{0}(t-k)+D \xi(t) \\
y(t)=C x(t)+E u_{0}(t-k) \\
y_{p}(t)=C_{p} x(t)+E u_{p} u_{0}(t-h) \\
z(t)=C x(t)+E u_{0}(t-k)+v(t)
\end{gathered}
$$

where $A, B, C, D, E, C_{p}, E_{p}$ are constant matrices. The delay free plant transfer of the linear subsystem, referred to above, may be written as $W_{0 k}=E+C \Phi B$, where $\Phi=(z I-A)^{-1}$. The input signal channels in the plant model are assumed to include a $k$-steps delay and the signals may be listed as: $x(t)=$ linear sub-system states; $u_{0}(t)=$ input signal to the linear subsystem; $u(t)=$ control signal; $y(t)=$ output signal; $z(t)=$ observations; $r(t)=$ set-point or reference; $y_{p}(t)=$ output to be controlled including cost-weighting; $r_{p}(t)=$ reference including cost-weighting. 


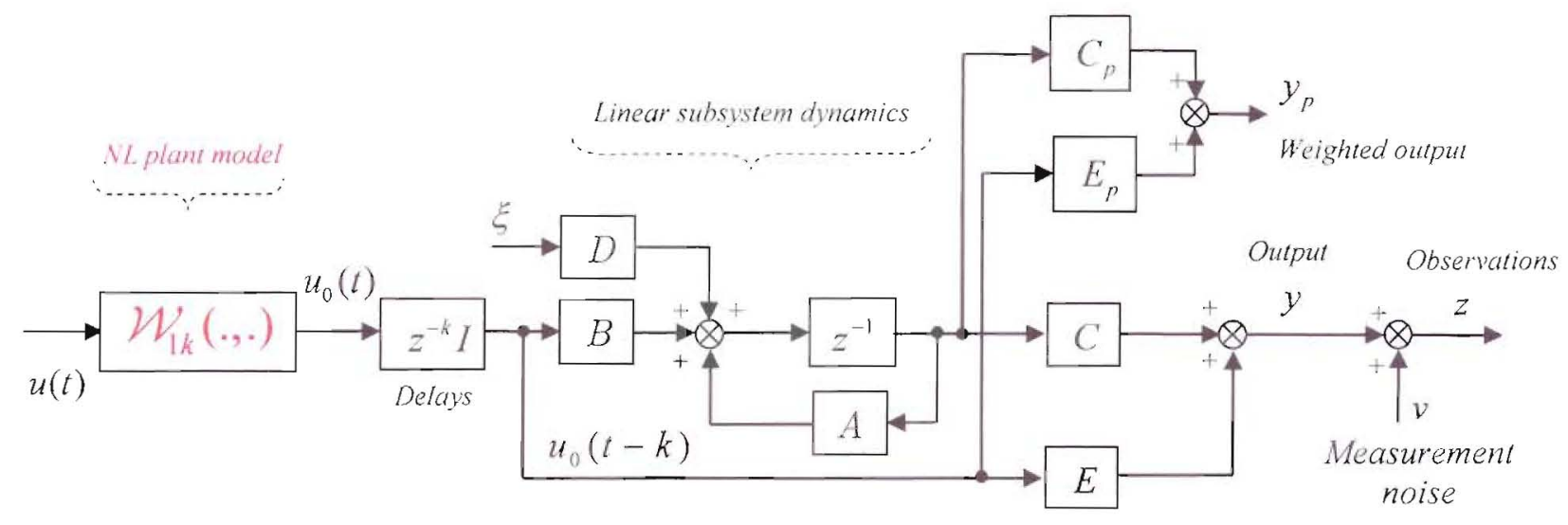

Fig. 2: Nonlinear Plant Input Subsystem and Linear Output and Disturbance Subsystem

Future Outputs and States: Future values of the states and outputs may be obtained as:

$$
x(t+i)=A^{\prime} x(t)+\sum_{j=1}^{1} A^{\prime-j}\left(B u_{0}(t+j-1-k)+D \xi(t+j-1)\right)
$$

The expression for the future states may be obtained by changing the time in (6) by the k-steps of the explicit transport delay giving:

$$
x(t+i+k)=A^{\prime} x(t+k)+\sum_{i=1}^{i} A^{\prime-1}\left(B u_{0}(t+j-1)+D \xi(t+j+k-1)\right)
$$

Future Weighted Outputs: The weighted output equation can include any stable dynamic costweighting $?_{p}(t)=P_{\mathrm{C}}\left(z^{-1}\right) y(t)$, which involves augmenting the state equation model. Noting (3) the weighted output $y_{p}(i)$ has the following form (for $i \geq 1$ ):

$$
y_{p}(t+i+k)=C_{p} A^{i} x(t+k)+\sum_{j=1}^{i} C_{p} A^{i-j}\left(B u_{0}(t+j-1)+D \xi(t+j+k-1)\right)+E_{p} u_{0}(t+i)
$$

The outputs are to be computed for controls in the interval $\tau \in[t, t+N]$. Introducing an obvious notation for these output signals they may be collected in an $N+1$ vector form as: 


$$
\begin{aligned}
& {\left[\begin{array}{c}
y_{p}(t+k) \\
y_{p}(t+1+k) \\
y_{p}(t+2+k) \\
\vdots \\
y_{p}(t+N+k)
\end{array}\right]=\left[\begin{array}{c}
C_{p} I \\
C_{p} A \\
C_{p} A^{2} \\
\vdots \\
C_{p} A^{N}
\end{array}\right] x(t+k)+\left[\begin{array}{cccc}
0 & 0 & \cdots & 0 \\
C_{p} B & 0 & \ddots & 0 \\
C_{p} A B & C_{p} B & & \\
\vdots & & \ddots & 0 \\
C_{p} A^{N-1} B & C_{p} A^{N-2} B & \cdots & C_{p} B
\end{array}\right]\left[\begin{array}{c}
u_{0}(t) \\
u_{0}(t+1) \\
\vdots \\
u_{0}(t+N-1)
\end{array}\right]} \\
& +\left[\begin{array}{c}
E_{p} u_{0}(t) \\
E_{p} u_{0}(t+1) \\
E_{p} u_{0}(t+2) \\
\vdots \\
E_{p} u_{0}(t+N)
\end{array}\right]+\left[\begin{array}{cccc}
0 & 0 & \cdots & 0 \\
C_{p} D & 0 & \ddots & 0 \\
C_{p} A D & C_{p} D & & \\
\vdots & & \ddots & 0 \\
C_{p} A^{N-1} D & C_{p} A^{N-2} D & \cdots & C_{p} D
\end{array}\right]\left[\begin{array}{c}
\xi(t+k) \\
\xi(t+1+k) \\
\vdots \\
\xi(t+N-1+k)
\end{array}\right]
\end{aligned}
$$

With an obvious definition of terms this equation may be written as:

$$
Y_{t+k, N}=C_{N} A_{N} x(t+k)+\left(C_{N} B_{N}+E_{N}\right) U_{t, N}^{0}+C_{N} D_{N} W_{t+k N}
$$

where the following vectors and block matrices may be defined for the case: $N>0$ :

$$
\begin{gathered}
C_{N}=\operatorname{diag}\left\{C_{p}, C_{p}, \ldots, C_{p}\right\} \quad \text { and } E_{N}=\operatorname{diag}\left\{E_{p}, E_{p}, \ldots, E_{p}\right\} \quad(N+I \text { square }) \\
A_{N}=\left[\begin{array}{c}
I \\
A \\
A^{2} \\
\vdots \\
A^{N}
\end{array}\right], \quad B_{N}=\left[\begin{array}{ccccc}
0 & 0 & \cdots & 0 & 0 \\
B & 0 & \cdots & 0 \\
\vdots & B & \ddots & \vdots \\
A^{N-1} B & A^{N-2} B & \cdots & B & 0
\end{array}\right], \quad D_{N}=\left[\begin{array}{cccc}
0 & 0 & \cdots & 0 \\
D & 0 & \ddots & 0 \\
A D & D & & \\
\vdots & & \ddots & 0 \\
A^{N-1} D & A^{N-2} D & \cdots & D
\end{array}\right] \\
W_{t, N}=\left[\begin{array}{c}
\xi(t) \\
\xi(t+1) \\
\vdots \\
\xi(t+N-1)
\end{array}\right], \quad U_{t, N}^{0}=\left[\begin{array}{c}
u_{0}(t) \\
u_{0}(t+1) \\
\vdots \\
u_{0}(t+N)
\end{array}\right], \quad R_{t, N}=\left[\begin{array}{c}
r_{p}(t) \\
r_{p}(t+1) \\
\vdots \\
r_{p}(t+N)
\end{array}\right]
\end{gathered}
$$


For the special case: $N=0, A_{N}=I, B_{N}=D_{N}=0, C_{N}=C_{p}, E_{N}=E_{p}$. The transfer $W_{t, N}$ denotes a vector of future white noise inputs and $U_{i . N}$ denotes a block vector of future control signals. The block vector $R_{t, N}$ denotes a vector of future reference signals that must include the same weighting as on the output (8) $r_{p}(t)=P_{c}\left(z^{-1}\right) r(t)$. The $k$ steps-ahead tracking error, that includes any dynamic error weighting, may be written as:

$$
E_{t+k, N}=R_{t+k, N}-Y_{t+k, N}=R_{t+k, N}-\left(C_{N} A_{N} x(t+k)+V_{N} U_{t, N}^{0}+C_{N} D_{N} W_{t+k, N}\right)
$$

The weighted inferred output to be minimised is assumed to have the same dimension as the control signal. The matrix $V_{N}$ in (12) for $N>0$, is of a block lower triangular form:

$$
V_{N}=C_{N} B_{N}+E_{N}=\left[\begin{array}{ccccc}
E_{p} & 0 & \cdots & 0 & 0 \\
C_{p} B & E_{p} & \cdots & \vdots & 0 \\
\vdots & C_{p} B & \ddots & & \vdots \\
C_{p} A^{N-2} B & \vdots & \ddots & E_{p} & 0 \\
C_{p} A^{N-1} B & C_{p} A^{N-2} B & \cdots & C_{p} B & E_{p}
\end{array}\right]
$$

For the special case of a single-stage $\operatorname{cost} N=0$ and this matrix must be defined as $V_{N}=E_{p}$.

\section{Prediction Model}

The i-steps ahead prediction of the output signal may be calculated by noting the above result ( 8 ) and assuming for the present that the future values of the control action are known. Thus let:

$$
\hat{y}_{p}(l+i+k \mid t)=E\left\{y_{p}(t+i+k) \mid t\right\}
$$


then,

$$
\hat{y}_{p}(t+i+k \mid t)=C_{p} A^{z} \hat{x}(t+k \mid t)+\sum_{j=1}^{i} C_{p} A^{i-j} B u_{0}(t+j-1)+E_{p} u_{0}(t+i)
$$

where $\hat{x}(t+k \mid t)$ denotes a least squares state estimate from a Kalman filter. Collecting results for the case $N>0$ the vector of predicted outputs $\hat{Y}_{t+k, N}$ may be obtained in the block matrix form:

$$
\left[\begin{array}{c}
\hat{y}_{p}(t+k \mid t) \\
\hat{y}_{p}(t+1+k \mid t) \\
\hat{y}_{p}(t+2+k \mid t) \\
\vdots \\
\hat{y}_{p}(t+N+k \mid t)
\end{array}\right]=\underbrace{\left[\begin{array}{c}
C_{p} I \\
C_{p} A \\
C_{p} A^{2} \\
\vdots \\
C_{p} A^{N}
\end{array}\right]}_{C_{N} A_{N}} \hat{x}(t+h \mid t)+\underbrace{\left[\begin{array}{ccccc}
E_{p} & 0 & \cdots & 0 & 0 \\
C_{p} B & E_{p} & \cdots & \vdots & 0 \\
\vdots & C_{p} B & \ddots & & \vdots \\
C_{p} A^{N-2} B & \vdots & \ddots & E_{p} & 0 \\
C_{p} A^{N-1} B & C_{p} A^{N-2} B & \cdots & C_{p} B & E_{p}
\end{array}\right]}_{V_{N}=C_{N} B_{N}+E_{N}} \underbrace{\left[\begin{array}{c}
u_{0}(t) \\
u_{0}(t+1) \\
\vdots \\
\vdots \\
u_{0}(t+N)
\end{array}\right]}_{U_{t, N}^{0}}
$$

This $N+1$ step-ahead prediction in (15) can clearly be written in the form:

$$
\hat{Y}_{t+k, N}=C_{N} A_{N} \hat{x}(t+k \mid t)+V_{N} U_{t, N}^{0}
$$

Output prediction error: $\quad \hat{Y}_{t+k, N}=Y_{t+k, N}-\hat{Y}_{i+k, N}$

$$
=C_{N} A_{N} x(t+k)+V_{N} U_{t, N}^{0}+C_{N} D_{N} W_{t+k, N}-\left(C_{N} A_{N} \hat{x}(t+k \mid t)+V_{N} U_{t, N}^{0}\right)
$$

Thence, the inferred output estimation error:

$$
\tilde{Y}_{t+k, N}=C_{N} A_{N} \tilde{x}(t+k i \mid t)+C_{N} D_{N} W_{t+k, N}
$$

where the $k$ steps-ahead state estimation error: $\tilde{x}(t+k i \mid t)=x(t+k i)-\hat{x}(t+k \mid t)$. The state estimation error is independent of the choice of control action. Also recall that the optimal $\hat{x}(t+k \mid t)$ and $\vec{x}(t+k \mid t)$ are orthogonal and the expectation of the product of the future values of the control action (assumed known in deriving the prediction equation), and the zero 
mean white noise driving signals, is null. It follows that the vector of predicted signals $\hat{Y}_{t+k, N}$ in (16) and the prediction error $\tilde{Y}_{t, N}$ are orthogonal.

\section{Kalman Estimator - Predictor Corrector Form}

The estimates are required from a Kalman filter summarised briefly as:

$$
\begin{array}{cc}
\hat{x}(t+1 \mid t)=A \hat{x}(t \mid t)+B u_{0}(t-k) & \text { (Predictor) } \\
\hat{x}(t+1 \mid t+1)=\hat{x}(t+1 \mid t)+K,(z(t+1)-\hat{z}(t+1 \mid t)) & \text { (Corrector) } \\
\hat{z}(t+1 \mid t)=C \hat{x}(t+1 \mid t)+E u_{0}(t+1-k) &
\end{array}
$$

The state estimate $\hat{x}(t+k \mid t)$ may be obtained, $k$ steps ahead, using a Kalman filter [11]. In this form of the estimator the number of states in the filter is not increased by the number of the synchronous delays $k$. The desired prediction equation:

$$
\hat{x}(t+k \mid t)=A^{k} \hat{x}(t \mid t)+T_{0}\left(k, z^{-1}\right) B u_{0}(t)
$$

where $T_{0}\left(k, z^{-1}\right)$ denotes a finite impulse response block, $T_{0}\left(0, z^{-1}\right)=I$ and for $k \geq 1$ :

$$
T_{0}\left(k, z^{-1}\right)=\left(I-A^{k} z^{-k}\right)(z I-A)^{-1}=z^{-1}\left(I+z^{-1} A+z^{-2} A^{2}+\ldots+z^{-k+1} A^{k-1}\right)
$$

Using (19) to (21) the optimal estimate may be written:

$$
\hat{x}(t+1 \mid t+1)=A \hat{x}(t \mid t)+B u_{0}(t-k)+K_{f}\left(z(t+1)-C A \hat{x}(t \mid t)-C B u_{0}(t-k)-E u_{0}(t+1-k)\right)
$$

The above equation may therefore be written:

$$
\hat{x}(t \mid t)=T_{f 1}\left(z^{-1}\right) z(t)+T_{12}\left(z^{-1}\right) u_{0}(t)
$$


where

$$
\begin{gathered}
T_{f 1}\left(z^{-1}\right)=\left(I-z^{-1}(I-K, C) A\right)^{-1} K_{f} \\
T_{f 2}\left(z^{-1}\right)=-\left(I-z^{-1}(I-K, C) A\right)^{-1} z^{-k}\left(K, E+\left(K_{j} C-I\right) B z^{-1}\right)
\end{gathered}
$$

Observe that for the Kalman filter to be unbiased the following equation must be satisfied:

$$
T_{f 1}\left(z^{-1}\right)\left(C \Phi\left(z^{-1}\right) B+E\right) z^{-k}+T_{f 2}\left(z^{-1}\right)=\Phi\left(z^{-1}\right) B z^{-k}
$$

This result may be verified using (25) and (26).

\section{Generalised Predictive Control Review}

A review of the derivation of the GPC controller is provided below where the input $\left(u_{0}\right)$ will be taken to be that for the linear sub-system. The GPC criterion [12] to be minimised:

$$
\left.J=E\left\{\sum_{j=0}^{N} \mathrm{e}_{p}(t+j+k)^{T} \mathrm{e}_{p}(t+j+k)+\lambda_{j}^{2} u_{0}(t+j)^{7} u_{0}(t+j)\right) \mid t\right\}
$$

where: $E\{. \mid t\}$ denotes the conditional expectation, conditioned on measurements up to time $t$ and $\lambda_{j}$ denotes a scalar control signal weighting factor. The vector of future weighted reference signal is denoted by $r_{p}(t+j+k)$ where the weighted error $\mathrm{e}_{p}(t)=r_{p}(t)-y_{p}(t)$. The future optimal control signal is to be calculated for the interval $\tau \varepsilon[t, t+N]$. The state-space models generating the signals $r_{p}$ and $y_{p}$ may include any dynamic cost-function weighting $P_{c^{\prime}}\left(z^{-1}\right),[11]$.

\section{Optimal Control Solution Using State Estimate Feedback}

It is not assumed that the states are available and hence an optimal state estimator must be introduced. The multi-step cost may then be written by using the above vectors and (29): 


$$
\begin{gathered}
J=E\{J,\}=E\left\{\left(R_{t+k, N}-Y_{t+k, N}\right)^{t}\left(R_{t+k, N}-Y_{i+k, N}\right)+U_{t, N}^{0 T} \Lambda_{N}^{2} U_{t, N}^{0} \mid t\right\} \\
=E\left\{\left(R_{t+k, N}-\left(\hat{Y}_{t+k, N}+\tilde{Y}_{t+k, N}\right)\right)^{T}\left(R_{t+k, N}-\left(\hat{Y}_{t+k, N}+\tilde{Y}_{t+k, N}\right)\right)+U_{t, N}^{0 T} \Lambda_{N}^{2} U_{t, N}^{0} \mid t\right\}
\end{gathered}
$$

where the cost weightings on the future inputs $u_{0}$ are written as $\Lambda_{N}^{2}=\operatorname{diag}\left\{\lambda_{0}^{2}, \lambda_{1}^{2}, \ldots, \lambda_{N}^{2}\right\}$. The terms in the cost-index can then be simplified, first by noting the optimal estimate $\hat{Y}_{t+k, N}$ is orthogonal to the estimation error $\tilde{Y}_{t+k_{i} N}$ and second by recalling the future reference $R_{t+k, N}$ is assumed to be a known signal over the $N+I$ steps. Simplifying, obtain the vector/matrix form:

Vector form GPC criterion: $\quad J=\left(R_{t+k, N}-\hat{Y}_{t+k, N}\right)^{T}\left(R_{t+k, N}-\hat{Y}_{1+k, N}\right)+U_{t, N}^{0 T} \Lambda_{N}^{2} U_{1, N}^{0}+J_{0}$

where $J_{0}=E\left\{\tilde{Y}_{r+k, N}^{T} \tilde{Y}_{t+k, N} \mid t\right\}$. Substituting from equation (16) for the vector of state-estimates:

$$
\hat{Y}_{t+k, N}=C_{N} A_{N} \hat{x}(t+k \mid t)+V_{N} U_{t, N}^{0}
$$

and writing:

$$
\tilde{R}_{t+k, N}=R_{t+k, N}-C_{N} A_{N} \hat{x}(t+k: t)
$$

Using these results the cost function may be expanded as:

$$
\begin{gathered}
J=\left(\tilde{R}_{t+k, N}-V_{N} U_{t, N}^{0}\right)^{T}\left(\tilde{R}_{t+k, N}-V_{N} U_{t, N}^{0}\right)+U_{t, N}^{0 T} \Lambda_{N}^{2} U_{t, N}^{0}+J_{0} \\
=\tilde{R}_{t+k, N}^{T} \tilde{R}_{t+k, N}-U_{t, N}^{0 T} V_{N}^{T} \tilde{R}_{t+k, N}-\tilde{R}_{t+k, N}^{T} V_{N} U_{t, N}^{0}+U_{t, N}^{0 T}\left(V_{N}^{T} V_{N}+\Lambda_{N}^{2}\right) U_{t, N}^{0}+J_{0}
\end{gathered}
$$

The procedure for minimising this cost term, if the signals are deterministic, is almost identical to that when the conditional cost is considered. The gradient of the cost-function must be set to zero, to obtain the vector of future optimal control signals. From a perturbation and gradient calculation [12], noting the $J_{0}$ term is independent of the control, the vector of future oplimal control signals:

$$
U_{t, N}^{0}=\left(V_{N}^{T} V_{N}+\Lambda_{N}^{2}\right)^{-1} V_{N}^{T}\left(R_{t+k, N}-C_{N} A_{N} \hat{l}(t+k \mid t)\right)
$$


The GPC optimal control is defined from this vector based on the receding horizon principle [13] and the optimal control is taken as the first element in the vector of future controls $U_{t, N}^{0}$.

\section{Equivalent Cost Optimisation Problem}

It is now shown that the above problem is equivalent to a special cost minimisation control problem which is needed to motivate the NPGMV problem introduced later. Let the constant positive definite, real symmetric matrix: $X_{N}=V_{N}^{T} V_{N}+\Lambda_{N}^{2}$ that enters the above solution, be

factorised into the form: $\quad Y^{T} Y=V_{N}^{T} V_{N}+\Lambda_{N}^{2}$

Observe that by completing the squares in equation (33) the cost-function may be written as:

$$
\begin{gathered}
J=\tilde{R}_{t+k, N}^{T} \tilde{R}_{t+k, N}-U_{t, N}^{0 T} V_{N}^{T} \tilde{R}_{t+k, N}-\tilde{R}_{t+k, N}^{T} V_{N} U_{t, N}^{0}+U_{t, N}^{0 T} Y^{T} Y U_{t, N}^{0}+J_{0} \\
=\left(\tilde{R}_{t+k, N}^{T} V_{N} Y^{-1}-U_{t+k, N}^{0 T} Y^{T}\right)\left(Y^{-T} V_{N}^{T} \tilde{R}_{t+k, N}-Y U_{t, N}^{0}\right)+\tilde{R}_{t+k, N}^{T}\left(I-V_{N} Y^{-1} Y^{-T} V_{N}^{T}\right) \tilde{R}_{t+k, N}+J_{0}
\end{gathered}
$$

That is, the cost-function:

$$
J=\hat{\Phi}_{t+k, N}^{T} \Phi_{t+k, N}+J_{10}(t)
$$

where the signal:

$$
\hat{\Phi}_{t+k, N}=Y^{-T} V_{N}^{T}\left(R_{t+k, N}-C_{N} A_{N} \hat{x}(t+k \mid t)\right)-Y U_{t, N}^{0}
$$

The terms that are independent of the control action may be written as: $J_{10}(t)=J_{0}+J_{1}(t)$ where,

$$
J_{1}(t)=\tilde{R}_{t+k, N}^{T}\left(I-V_{N} Y^{-1} Y^{-T} V_{N}^{T}\right) \tilde{R}_{t+k, N}
$$

Since the last term $J_{10}(t)$ in equation (36) does not depend upon control action the optimal control is found by setting the first term to zero, giving the same control as defined in (34). It follows that the GPC optimal controller for the above linear system is the same as the controller to minimise the Euclidean norm of the signal $\hat{\Phi}_{t+k, N}$ in (37). 
The above motivates the definition of a new multi-step minimum variance cost problem that has the same solution for the optimal controller that is needed before the nonlinear problem can be considered. A new signal to be minimised involving a weighted sum of error and inputs:

$$
\phi=P_{c} e+F_{\mathrm{c} 0} u_{0}
$$

The vector of future values of this signal, for a multi-step cost index, may therefore be written as:

$$
\Phi_{t, \lambda}=P_{C, N} E_{i, N}+F_{C N}^{0} U_{T, N}^{0}
$$

Introduce cost-function weightings, based on the $G M V$ weightings above, to have the form:

$$
P_{C N}=V_{N}^{T} \quad \text { and } \quad F_{C N}^{0}=-\Lambda_{N}^{2} \text {. }
$$

which will be justified by the property established in Theorem 3.1 below. Then motivated by the preceding, define a new minimum variance multi-step cost-function, using a vector of signals:

$$
\tilde{J}=E\left\{\tilde{J}_{t}\right\}=E\left\{\Phi_{t+k, N}^{T} \Phi_{t+k, N} \mid t\right\}
$$

Predicting forward $k$-steps

$$
\Phi_{t+k, N}=P_{c, N}\left(R_{t+k, N}-Y_{t+k, N}\right)+F_{c i N}^{0} U_{t, N}^{0}
$$

Consider the signal $\Phi_{t+k, N}$ and substitute for the outputs $Y_{t+k, N}=\hat{Y}_{t+k, N}+\tilde{Y}_{t+k, N}$. Then from (43):

$$
\Phi_{t+k, N}=\left(P_{c, N}\left(R_{t+k, N}-\hat{Y}_{t+k, N}\right)+F_{C N}^{0} U_{t, N}^{0}\right)-P_{C N} \tilde{Y}_{i+k, N}
$$

This expression may be written in terms of the estimate and the estimation error vector as:

$$
\Phi_{t+k, N}=\hat{\Phi}_{t+k, N}+\tilde{\Phi}_{t+k, N}
$$

where the predicted signal: $\quad \hat{\Phi}_{t+k, N}=\left(P_{c N}\left(R_{t+k, N}-\hat{Y}_{t+k, N}\right)+F_{c N}^{0} U_{t, N}^{0}\right)$ 
and the prediction error:

$$
\tilde{\Phi}_{t+k, N}=-P_{C N} \tilde{Y}_{t+k, N}
$$

Multi-Step Cost Index: The performance index (42) may therefore be simplified and written as:

$$
\tilde{J}=E\left\{\tilde{J}_{t}\right\}=E\left\{\Phi_{t+k, N}^{T} \Phi_{t+k, N} \mid t\right\}=E\left\{\left(\hat{\Phi}_{t+k, N}+\tilde{\Phi}_{t+k, N}\right)^{T}\left(\hat{\Phi}_{i+k, N}+\tilde{\Phi}_{t+k, N}\right) \mid t\right\}
$$

The terms in (42) can be simplified, recalling the optimal estimate $\hat{Y}_{t+k, N}$ and the estimation error $\tilde{Y}_{t+k, N}$ are orthogonal, and the future reference trajectory $R_{t+k, N}$ is a known signal. Thus,

$$
\begin{gathered}
\tilde{J}=E\left\{\tilde{\Phi}_{t+k, N}^{T} \tilde{\Phi}_{t+k, N} \mid t\right\} \\
+E\left\{\tilde{\Phi}_{t+k, N}^{T} \tilde{\Phi}_{t+k, N} \mid t\right\}+E\left\{\tilde{\Phi}_{t+k, N}^{T} \tilde{\Phi}_{t+k, N} \mid t\right\}+E\left\{\tilde{\Phi}_{t+k, N}^{T} \tilde{\Phi}_{t+k, N} \mid t\right\} \\
=\hat{\Phi}_{t+k, N}^{T} \hat{\Phi}_{t+k, N}+E\left\{\tilde{\Phi}_{t+k, N}^{T} \tilde{\Phi}_{t+k, N} \mid t\right\}
\end{gathered}
$$

Thence, the cost-function may be written: $\quad \tilde{J}(t)=\hat{\Phi}_{t+k_{i, N}}^{T} \hat{\Phi}_{t+k_{1} N}+\tilde{J}_{1}(t)$

The last cost term in (48) is independent of control action and may be written as:

$$
\tilde{J}_{1}(t)=E\left\{\tilde{\Phi}_{t+k, N}^{T} \tilde{\Phi}_{t+k, N} \mid t\right\}=E\left\{\tilde{Y}_{t+k, N}^{T} P_{C N}^{T} P_{C N} \tilde{Y}_{t+k, N} \mid t\right\}
$$

This vector $\hat{\Phi}_{t+k, N}$ may be simplified by substituting for $\hat{Y}_{t+k, N}$, from (16) and (35):

$$
\hat{\Phi}_{t+k, N}=P_{C N}\left(R_{t+k, N}-\hat{Y}_{t+k, N}\right)+F_{C N}^{0} U_{t, N}^{0}=P_{C N}\left(R_{t+k, N}-C_{N} A_{N} \hat{x}(t+k \mid t)\right)-\left(V_{N}^{T} V_{N}+\Lambda_{N}^{2}\right) U_{t, N}^{0}
$$

Thence, from (35) obtain: $\quad \hat{\Phi}_{t+k, N}=P_{C N}\left(R_{t+k, N}-C_{N} A_{N} \hat{x}(t+k \mid t)\right)-X_{N} U_{t, N}^{0}$

Recall the optimal multi-step minimum variance predictive control sets the first squared term in (48) to zero $\hat{\Phi}_{t+k, N}=0$. The resulting control is the same as the vector of future GPC controls. 


\section{Theorem 3.1: Equivalent Minimum Variance Cost Optimisation Problem}

Consider the minimisation of the GPC cost index (28) for the system and assumptions introduced in $\S 2$, where the nonlinear subsystem: $\mathcal{W}_{1 \mathrm{k}}=I$ and the vector of optimal GPC controls is given by (34). If the cost index is redefined to have a multi-step minimum variance form (42) $\tilde{J}(t)=E\left\{\Phi_{t+k, N}^{T} \Phi_{t+k, N} \mid t\right\}$, where $\Phi_{t+k, N}=P_{C N}\left(R_{t+k, N}-Y_{t+k, N}\right)+F_{C N}^{0} U_{t, N}^{0}$ and the cost-function weightings $P_{C N}=V_{N}^{T}$ and $F_{C N}^{0}=-\Lambda_{N}^{2}$, then the vector of future optimal controls is identical to the GPC controls in (34).

Solution: The proof follows by collecting the results in the section above.

\section{Nonlinear Predictive GMV Control}

The actual input to the system is the control signal $u(t)$, shown in Fig. 1, rather than the input to the linear sub-system $u_{0}$. The cost-function for the nonlinear control problem of interest must therefore include an additional control signal costing term, although the costing on the intermediate signal $u_{0}(t)$ can be retained to examine limiting cases. If the smallest delay in each output channel of the plant is of magnitude $k$-steps this implies the control affects the output $k$ steps later and the control costing should include the delay $\left(\mathcal{F}_{c} u\right)(t)=z^{-k}\left(\mathcal{F}_{c k} u\right)(t)$. This weighting on the nonlinear sub-system input will be assumed to be full rank and invertible and can be a linear dynamic operator but it may also be chosen to be nonlinear [11]. Thus, consider a new signal to be minimised: $\quad \phi_{0}(t)=P_{c} e(t)+F_{\mathrm{c} 0} u_{0}(t)+\left(\mathcal{F}_{c} u\right)(t)$

A multi-step cost index may now be defined that is an extension of the cost-function in (42).

Extended Multi-Step Cost Funcrion: $\quad J_{p}=E\left\{\Phi_{t+k, N}^{0 T T} \Phi_{t+k, N}^{0} \mid t\right\}$ 
The signal $\Phi_{t+k, N}^{0}$ is defined to include the future control signal costing terms:

$$
\Phi_{t+k, N}^{0}=P_{c, N} E_{t+k, N}+F_{c N}^{0} U_{t, N}^{0}+\left(\mathcal{F}_{c k, N} U_{t, N}\right)=P_{c, N}\left(R_{t+k, N}-Y_{t+k, N}\right)+F_{c N}^{0} U_{t, N}^{0}+\mathcal{F}_{\mathrm{c} k, N} U_{t, N}
$$

where the non-linear function $\mathcal{F}_{\mathrm{c} k, N} U_{t, N}$ will normally be defined to have a simple diagonal form:

$$
\mathcal{F}_{c k, N} U_{t, N}=\operatorname{diag}\left\{\left(\mathcal{F}_{c k} u\right)(t),\left(\mathcal{F}_{c k} u\right)(t+1), \ldots,\left(\mathcal{F}_{c k} u\right)(t+N)\right\}
$$

and the vector of inputs: $U_{t, N}^{0}=\left(\mathcal{W}_{1 \mathrm{k}, \mathrm{N}} U_{t, N}\right)$, where $\mathcal{W}_{1 \mathrm{k}, \mathrm{N}}$ also has a block diagonal matrix form:

$$
\mathcal{W}_{1 k, N} U_{t, N}=\operatorname{diag}\left\{\mathcal{W}_{1 k}, \mathcal{W}_{1 k}, \ldots, \mathcal{W}_{1 k}\right\} U_{t, N}=\left[\left(\mathcal{W}_{1, k} u\right)(t)^{T}, \ldots,\left(\mathcal{W}_{1 k} u\right)(t+N)^{T}\right]^{T}
$$

The problem simplifies when $N=0$ to the single-step $N G M V$ control problem.

\section{The NPGMV Control Problem Solution}

The solution follows from very similar steps to those in $\$ 3.3$ and will therefore be summarised briefly. Observe from (43) that $\Phi_{t, N}^{0}=\Phi_{t, N}+z^{-k} \mathcal{F}_{c k, N} U_{t, N}$ and $\Phi_{t+k, N}^{0}=\hat{\Phi}_{t+k, N}^{0}+\tilde{\Phi}_{t+k, N}^{0}$ where

$$
\Phi_{i+k, N}^{0}=\hat{\Phi}_{t+k, N}+\mathcal{F}_{\mathrm{c} k, N} U_{t, N}=P_{c i s}\left(R_{t+k, N}-\hat{Y}_{t+k, N}\right)+F_{c i N}^{0} U_{t, N}^{0}+\mathcal{F}_{\mathrm{c} k, N} U_{t, N}
$$

The estimation error:

$$
\tilde{\Phi}_{t+k, N}^{0}=\tilde{\Phi}_{t+k, N}=-V_{N}^{T} \tilde{Y}_{t+k, N}
$$

The future predicted values in the signal: $\hat{\Phi}_{t+k, N}^{0}$ involves the estimated vector of weighted outputs $\hat{Y}_{l+k, N}$ and these are orthogonal to $\tilde{Y}_{l+k, N}$. Note the estimation error is zero mean and the expected value of the product with any known signal is null. The cost-function may therefore be written as:

$$
\tilde{J}(t)=\hat{\Phi}_{t+k, N}^{0 T} \hat{\Phi}_{t+k, N}^{0}+\tilde{J}_{1}(t)
$$

where the optimal control sets $\Phi_{t+k, N}^{0}=0$. The condition for optimality becomes: 


$$
P_{C N}\left(R_{t+k, N}-\hat{Y}_{t+k, N}\right)+\left(\mathcal{F}_{\mathrm{c} k, N}+F_{C N}^{0} \mathcal{W}_{1 \mathrm{k}, N}\right) U_{t, N}=0
$$

\section{NPGMV Optimal Control}

The vector of future controls follows from the condition for optimality in (59):

$$
U_{t, N}=-\left(\mathcal{F}_{\mathrm{c} k, N}-\Lambda_{N}^{2} \mathcal{W}_{1 \mathrm{k}, \mathrm{N}}\right)^{-1} P_{c, N}\left(R_{t+k, N}-\hat{Y}_{t+k, N}\right)
$$

A solution of (59), that is useful for implementation becomes:

$$
U_{t, N}=-\mathcal{F}_{\mathrm{c} k, N}^{-1}\left(P_{c, N}\left(R_{t+k, N}-\hat{Y}_{t+k, N}\right)-\Lambda_{N}^{2} \mathcal{W}_{1 \mathrm{k}, N} U_{t, N}\right)
$$

The optimal predictive control law is nonlinear, since it involves the nonlinear control signal costing term $\mathcal{F}_{c k, N}$ and the nonlinear model for the plant $\mathcal{W}_{\mathbf{l k}, \mathrm{N}}$. Further simplification is possible by substituting from (16) for $\hat{Y}_{t+k, N}$, since equation (59) may be written as:

or

$$
\begin{gathered}
P_{c, N}\left(R_{t+k_{s}, N}-\left(C_{N} A_{N} \hat{x}(t+k \mid t)+V_{N} U_{t, N}^{0}\right)\right)+\left(\mathcal{F}_{\mathrm{c} k, N}+F_{c N}^{0} \mathcal{W}_{\mathrm{lk}, N}\right) U_{t, N}=0 \\
P_{c, N}\left(R_{t+k, N}-C_{N} A_{N} \hat{x}(t+k \mid t)\right)+\left(\mathcal{F}_{\mathrm{c} k, N}-X_{N} \mathcal{W}_{1 \mathrm{k}, N}\right) U_{t, N}=0
\end{gathered}
$$

This condition for optimality is the equivalent of that stemming from $(50)$ but with the $\mathcal{F}_{\mathrm{c} k, N}$ is added. Two alternative solutions for the vector of future optimal controls, in terms of the estimate of the future predicted state, therefore become:

or

$$
\begin{gathered}
U_{t, N}=-\left(\mathcal{F}_{c k, N}-X_{N} \mathcal{W}_{1 k, N}\right)^{-1} P_{c N}\left(R_{t+k, N}-C_{N} A_{N} \hat{x}(t+k \mid t)\right) \\
U_{t, N}=-\mathcal{F}_{c k, N}^{-1}\left(P_{c, N}\left(R_{t+k, N}-C_{N} A_{N} \hat{x}(t+k ; \mid t)\right)-X_{N} \mathcal{W}_{1 \mathrm{k}, N} U_{t, N}\right)
\end{gathered}
$$

Remarks: The control law is implemented using a receding horizon philosophy and it becomes identical to the GPC controller (34) in the limiting linear case when the control costing tends to 
zero $\left(\mathcal{F}_{c k, N} \rightarrow 0, \mathcal{W}_{1 k, N}=I\right)$. From (63) if the control weighting $\mathcal{F}_{c k, N} \rightarrow 0$ then $U_{t, N}$ will introduce the inverse of the plant model $\mathcal{W}_{1 \mathrm{k}, \mathrm{N}}$ (if one exists) and the resulting vector of future controls $U_{t, N}^{0}$ will then be the same as the GPC controls for the linear system that remains.

\section{Optimal Nonlinear Predictive Control Signal}

These expressions can be simplified further by substituting for the expression for the optimal predicted state in (22) and invoking the condition for optimality in (62):

$$
P_{c N}\left(R_{t+k, N}-C_{N} A_{N}\left(A^{k} \hat{x}(t \mid t)+T_{0}\left(k, z^{-1}\right) B u_{0}(t)\right)\right)+\left(\mathcal{F}_{\mathrm{c} k, N}-X_{N} \mathcal{W}_{\mathrm{lk}, \mathrm{N}}\right) U_{t, N}=0
$$

Recall $u_{0}(t)=\mathcal{W}_{1 \mathrm{k}} u(t)$ and write: $\quad \tilde{R}_{t+k, N}^{0}=R_{t+k, N}-C_{N} A_{N} A^{k} \hat{x}(t \mid t)$

The alternative form for the condition for optimality follows as:

$$
P_{c N}\left(\tilde{R}_{t+k, N}^{0}-C_{N} A_{N} T_{0}\left(k, z^{-1}\right) B\left(\mathcal{W}_{1 \mathrm{k}} u\right)(t)\right)+\left(\mathcal{F}_{\mathrm{ck}, N}-X_{N} \mathcal{W}_{1 \mathrm{k}, N}\right) U_{t, N}=0
$$

giving

$$
U_{t, N}=-\left(\mathcal{F}_{\mathrm{c} k, N}-X_{N} \mathcal{W}_{1 \mathrm{k}_{, N}}\right)^{-1} P_{c N}\left(\tilde{R}_{t+k, N}^{0}-C_{N} A_{N} T_{0}\left(k, z^{-1}\right) B\left(\mathcal{W}_{1 \mathrm{k}} u\right)(t)\right)
$$

An alternative expression also follows from (66):

$$
U_{t, N}=-\mathcal{F}_{\mathrm{c} k, N}^{-1}\left(P_{C N}\left(\tilde{R}_{t+k, N}^{0}-C_{N} A_{N} T_{0}\left(k, z^{-1}\right) B\left(\mathcal{W}_{1 \mathrm{k}} u\right)(t)\right)-X_{N} \mathcal{W}_{1 \mathrm{k}, N} U_{t, N}\right)
$$

To simplify the equations also introduce the constant matrix:

$$
\mathrm{C}_{\phi}=P_{C N} C_{N} A_{N}=V_{N}^{T} C_{N} A_{N}
$$

Condition for optimality: Equation (66) may be written as:

$$
P_{C N N} \vec{R}_{t+k, N}^{0}+\left(\mathcal{F}_{\mathrm{c} k, N}-X_{N} \mathcal{W}_{1 \mathrm{k}, \mathrm{V}}-\mathrm{C}_{\phi} T_{0}\left(k, z^{-1}\right) B C_{10} \mathcal{W}_{1 \mathrm{k}, \mathrm{N}}\right) U_{t, N}=0
$$


Optimal control: Two possible expressions for the vector of future optimal controls follow:

$$
\begin{gathered}
U_{t, N}=-\left(\mathcal{F}_{\mathrm{c} k, N}-X_{N} \mathcal{W}_{1 \mathrm{k}, \mathrm{N}}-\mathrm{C}_{\phi} T_{0}\left(k, z^{-1}\right) B C_{I 0} \mathcal{W}_{1 \mathrm{k}, N}\right)^{-1} P_{C N} \tilde{R}_{t+k, N}^{0} \\
\text { or } \quad U_{t, N}=-\mathcal{F}_{\mathrm{c} k, N}^{-1}\left(P_{C N}\left(R_{t+k, N}-C_{N} A_{N} A^{k} \hat{x}(t \mid t)\right)-\left(X_{N}+C_{\phi} T_{0}\left(k, z^{-1}\right) B C_{10}\right) \mathcal{W}_{1 \mathrm{k}, N} U_{t, N}\right)
\end{gathered}
$$

\section{Theorem 4.1: NPGMV Optimal Controller}

Consider the linear components of the plant, disturbance and output weighting models in state space form (2), (3) with input from the nonlinear finite gain stable plant dynamics $\mathcal{W}_{1 k}$. The multi-step predictive control cost-function to be minimised, involving a sum of future cost terms, is defined in vector form as:

$$
J_{p}=E\left\{\Phi_{t+k, N}^{0 T} \Phi_{t+k, N}^{0} \mid t\right\}
$$

where $N>0$ and the signal $\Phi_{t+k, N}^{0}$ depends upon future error, input and nonlinear control signal costing terms:

$$
\Phi_{t+k, N}^{0}=P_{c N} E_{t+k, N}+F_{c N N}^{0} U_{t, N}^{0}+\mathcal{F}_{c k, N} U_{t, N}
$$

The error and input cost-function weightings are introduced as in the GPC problem (28) and these determine the block matrix cost forms $P_{C N}=V_{N}^{T}$ and $F_{C N}^{0}=-\Lambda_{N}^{2}$. The control cost weighting may be nonlinear and has the form $\left(\mathcal{F}_{c} u\right)(t)=\left(\mathcal{F}_{c k} u\right)(t-k)$, where $k$ represents the transport delay and $\mathcal{F}_{c k}$ is full rank and invertible. Define the constant matrix $X_{N}=V_{N}^{T} V_{N}+\Lambda_{N}^{2}$ then the NPGMV optimal control law to minimize the variance $(73)$ is given as:

$$
U_{t, N}=-\left(\mathcal{F}_{\mathrm{c} k, N}-\left(X_{N}+\mathrm{C}_{\phi} T_{0}\left(k, z^{-1}\right) B\right) \mathcal{W}_{1 \mathrm{k}, N}\right)^{-1} P_{C N}\left(R_{t+k, N}-C_{N} A_{N} A^{k} \hat{x}(t \mid t)\right)
$$


The current control can be computed using the receding horizon principle from the first element in the alternative expression for the vector of future optimal controls:

$$
U_{t, N}=-\mathcal{F}_{\mathrm{c} k, N}^{-1}\left(\left(P_{\mathrm{c} N} R_{t+k, N}-C_{\phi} A^{k} \hat{x}(t \mid t)\right)-\left(X_{N}+\mathrm{C}_{\phi} T_{0}\left(k, z^{-1}\right) B C_{10}\right) \mathcal{W}_{1 \mathrm{k}, N} U_{t, N}\right)
$$

and the finite impulse response term $T_{0}\left(k, z^{-1}\right)=\left(I-A^{k} z^{-k}\right)(z l-A)^{-1}$ and matrix $C_{\phi}=P_{C N} C_{N} A_{N}$.

Solution: The proof of the optimal control was given before the Theorem and the assumption to ensure closed loop stability is explained in the stability analysis below.

Remarks: The expression (76) leads to the structure in Fig. 3 which is useful for implementation. This involves a Kalman predictor stage and note that the order of the Kalman filter depends only on the delay free linear subsystems and not the channel delays. If the output weighting $P_{c}$ includes a near integrator it appears in the feedback and reference channels and it is desirable to move this integrator term into the common path. The cost-index (74) when the input costing $F_{C N}^{0}$ is null gives $\Phi_{t+k, N}^{0}=P_{c N} E_{t+k, N}+\left(\mathcal{F}_{c k, N} U_{t, N}\right)=V_{N}^{T} E_{t+k, N}+\left(\mathcal{F}_{c k, N} U_{t, N}\right)$ but for a single-step cost $V_{N}=E_{p}$ and in this case $\Phi_{i+k, N}^{0}=E_{p}^{T} E_{t+k, N}+\left(\mathcal{F}_{\mathrm{c} k, N} U_{t, N}\right)$. The limiting case of the NPGMV controller is therefore related to an $N G M V$ controller where the error weighting is scaled by the $E_{p}^{T}$ term.

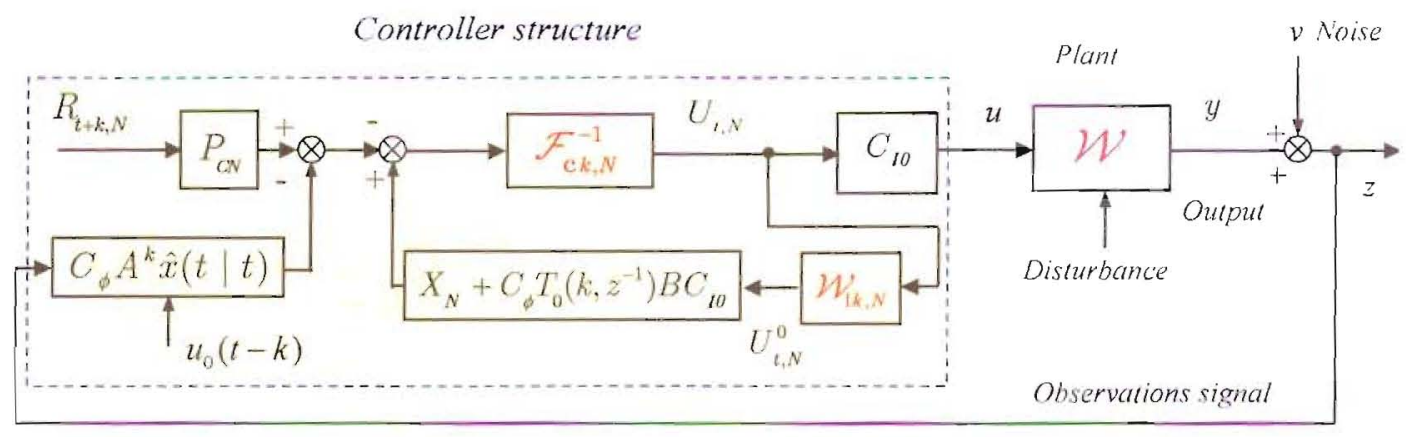

Fig. 3: NPGMV Current Control Signal Generation and Kalman Predictor 


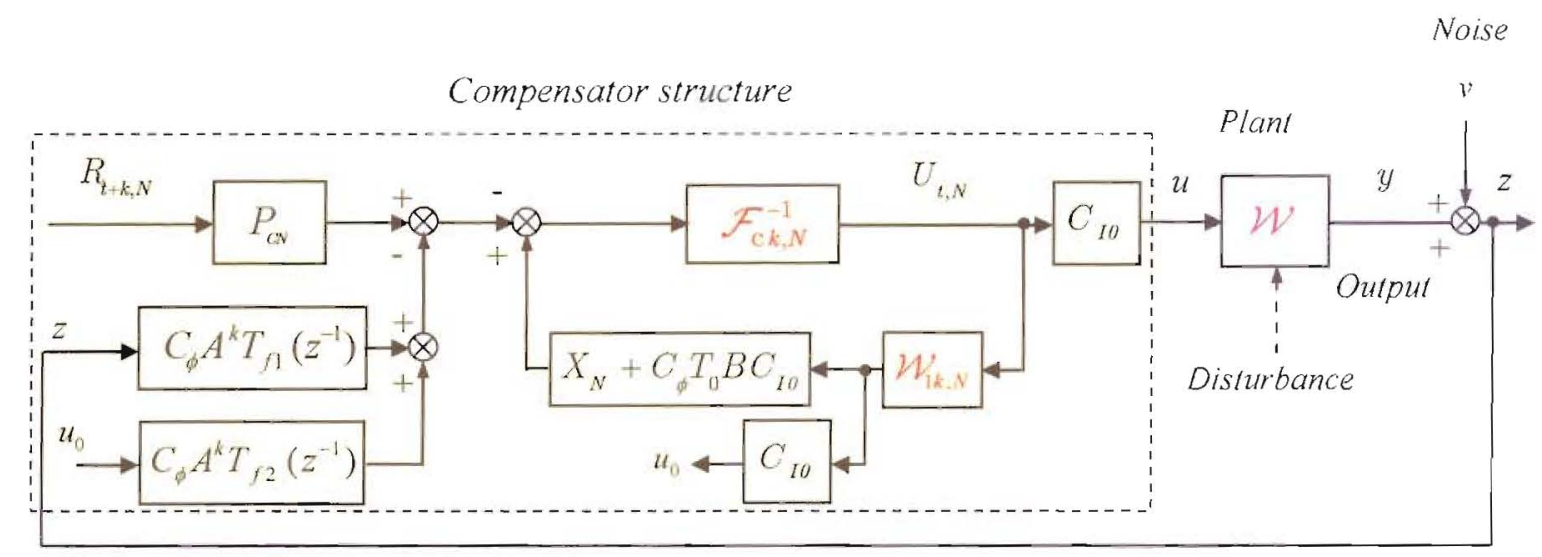

Fig. 4: NPGMV Compensator in Estimation Future Controls Form

\section{Stability of the Closed Loop and Design Issues}

To consider stability properties a different expression is required for the control action where the results are expressed in closed loop operator form. An algebraic result is first required involving terms from the Kalman filter equations. Recall from (27):

$$
C_{\phi} A^{k} T_{f 1}(C \Phi B+E) z^{-k}+C_{\phi} A^{k} T_{f 2}=C_{\phi} A^{k} \Phi B z^{-k}
$$

but from (23) $T_{0}\left(k, z^{-1}\right)=\left(I-A^{k} z^{-k}\right)(z I-A)^{-1}$. Using these results and noting $\mathrm{W}_{0 k}=E+C \Phi B$, the desired result is obtained as:

$$
C_{\phi} T_{0}\left(k, z^{-1}\right) B+C_{\phi} A^{k} T_{f 2}\left(z^{-1}\right)+C_{\phi} A^{k} T_{f 1}\left(z^{-1}\right) z^{-k} W_{0 k}=C_{\phi} \Phi\left(z^{-1}\right) B
$$

\section{Assumptions and Closed Loop Expressions}

For linear $G M V$ designs stability is ensured when the combination of a control weighting and an error weighted plant model transfer is strictly minimum phase. For the proposed nonlinear predictive control it is shown below that a nonlinear operator $\left(I-\mathcal{F}_{c k, N}^{-1}\left(X_{N}+C_{\phi} \Phi B C_{\mathrm{I} 0}\right) \mathcal{W}_{1 \mathrm{k}, \mathrm{N}}\right)$ must have a stable inverse where the measure of stability, such as finite gain stability, depends 
upon the assumption of stability on the nonlinear plant sub-system $\mathcal{W}_{1 k}$. For this stability discussion assume that the stochastic inputs are null. Then $z(t)=C x(t)+E u_{0}(t-k)+v(t)$

$\rightarrow(E+C \Phi B) u_{0}(t-k)$, and the optimal control becomes:

$$
\begin{gathered}
U_{t,}=-\mathcal{F}_{\mathrm{c} k, N}^{-1}\left(P_{c} R_{t+k, N}-C_{\phi} A^{k} T_{f 1}\left(z^{-1}\right) W_{0 k} u_{0}(t-k)-C_{\phi} A^{k} T_{f 2}\left(z^{-1}\right) u_{0}(t)\right. \\
\left.-\mathrm{C}_{\phi} T_{0}\left(k, z^{-1}\right) B u_{0}(t)-X_{N} W_{1 \mathrm{k} . N} U_{t, N}\right)
\end{gathered}
$$

Substituting from (77):

$$
\begin{gathered}
U_{1}=-\mathcal{F}_{\mathrm{c} k, N}^{-1}\left(P_{1} R_{t+k, N}-X_{N} W_{1 \mathrm{k}, N} U_{1 N}\right. \\
\left.-C_{\phi} A^{k} T_{11}\left(z^{-1}\right) W_{0 k} u_{0}(t-k)-C_{\phi} A^{k} T_{, 2}\left(z^{-1}\right) u_{0}(t)-C_{\phi} T_{0}\left(k, z^{-1}\right) B u_{0}(t)\right) \\
=-\mathcal{F}_{\mathrm{c} k, N}^{-1}\left(P_{C N} R_{t+k, N}-X_{N} W_{1 \mathrm{k}, N} U_{t, N}-C_{\phi} \Phi B u_{0}(t)\right)
\end{gathered}
$$

The control costing is normally a linear model and under this assumption (78) corresponds to the following condition for optimality:

$$
\left(I-\mathcal{F}_{c k, N}^{-1}\left(X_{N}+C_{\phi} \Phi B C_{\mathrm{I} 0}\right) \mathcal{W}_{1 \mathrm{k}, N}\right) U_{t, N}=-\mathcal{F}_{\mathrm{ck}, N}^{-1} P_{C N} R_{t+k, N}
$$

where $\left(\mathcal{W}_{1 \mathrm{k}, \mathrm{N}} U_{t, N}\right)=\left[\left(\mathcal{W}_{1 k} u\right)(t)^{T}, \ldots,\left(\mathcal{W}_{1 k} u\right)(t+N)^{T}\right]^{T}$. The desired expressions for the vectors of future optimal controls and nonlinear plant subsystem outputs therefore become:

\section{Future controls:}

$$
U_{t, N}=\left(I-\mathcal{F}_{\mathrm{c} k, N}^{-1}\left(X_{N}+C_{\phi} \Phi B C_{\mathrm{I} 0}\right) \mathcal{W}_{1 \mathrm{k}, \mathrm{N}}\right)^{-1}\left(-\mathcal{F}_{\mathrm{c} k, N}^{-1} P_{c, N} R_{t+k, N}\right)
$$




\section{NL Subsystem future outputs:}

$$
\mathcal{W}_{\mathrm{k}, \mathrm{N}} U_{I, N}=\mathcal{W}_{\mathrm{k}, N}\left(I-\mathcal{F}_{\mathrm{c} k, N}^{-1}\left(X_{N}+C_{\phi} \Phi B C_{10}\right) \mathcal{W}_{1 \mathrm{k}, \mathrm{N}}\right)^{-1}\left(-\mathcal{F}_{\mathrm{c} k, N}^{-1} P_{C,} R_{I+k, N}\right)
$$

\section{Total NL future plant outputs:}

$$
\mathcal{W}_{k, N} U_{t, N}=\mathcal{W}_{\mathrm{k}, N}\left(I-\mathcal{F}_{\mathrm{ck}, N}^{-1}\left(X_{N}+C_{\phi} \Phi B C_{10}\right) \mathcal{W}_{\mathrm{ik}, \mathrm{N}}\right)^{-1}\left(-\mathcal{F}_{\mathrm{c} k, N}^{-1} P_{C, N} R_{t+k, N}\right)
$$

\section{Stability Condition and Cost Function Weightings}

The operator $\left(I-\mathcal{F}_{\mathrm{c} k, N}^{-1}\left(X_{N}+C_{\phi} \Phi B C_{\mathrm{I} 0}\right) \mathcal{W}_{1 \mathrm{k}, \mathrm{N}}\right)^{-1}$ is assumed to be finite gain $m_{2}$ stable and if the linear plant sun-system is open loop stable all the operators in (80) to (82) are stable. If the open loop plant is not stable then further assumptions are needed to guarantee the stability of terms in (82).

If there exists a PID controller that will stabilize the nonlinear system, without transport delay elements, then a set of cost weightings can be defined to guarantee the existence of this inverse and hence ensure the stability of the closed-loop. Assume that only the error and control weightings are used, and that the input weighting $\Lambda_{N}^{2} \rightarrow 0$. Then $X_{N}=V_{N}^{T} V_{N}+\Lambda_{N}^{2} \rightarrow V_{N}^{T} V_{N}$

$$
\begin{aligned}
& U_{t, N}=\left(I-\mathcal{F}_{\mathrm{c} k, N}^{-1}\left(X_{N}+C_{\phi} \Phi B C_{\mathrm{l} 0}\right) \mathcal{W}_{1 \mathrm{k}, N}\right)^{-1}\left(-\mathcal{F}_{\mathrm{ck}, N}^{-1} P_{c N} R_{t+k, N}\right) \\
& \rightarrow\left(I-\mathcal{F}_{\mathrm{c} k, N}^{-1} V_{N}^{T}\left(V_{N}+C_{N} A_{N} \Phi B C_{\mathrm{I} 0}\right) \mathcal{W}_{1 \mathrm{k}, N}\right)^{-1}\left(-\mathcal{F}_{\mathrm{c} k, N}^{-1} P_{c, N} R_{t+k, N}\right)
\end{aligned}
$$

Recall that in the single-step cost problem, where $N=0$, the matrix $V_{N}=E_{p}$ is assumed square and non-singular. Hence $u(t) \rightarrow\left(I-\mathcal{F}_{c k}^{-1} E_{p}^{T}\left(E_{p}+C_{p} \Phi B\right) \mathcal{W}_{\mathrm{lk}}\right)^{-1}\left(-\mathcal{F}_{\mathrm{ck}}^{-1} E_{p}^{T} r_{p}(t)\right)$. Also assume the 
dynamic weighting is on the plant outputs $y_{p}(t)=P_{c}\left(z^{-1}\right) y(t)$ then $E_{p}+C_{p} \Phi B=P_{c}\left(z^{-1}\right) W_{0 k}$. It follows that in this limiting case: $\quad u(t) \rightarrow\left(I-\mathcal{F}_{c k}^{-1} E_{p}^{T} P_{c} W_{0 k} W_{1 \mathrm{k}}\right)^{-1}\left(-\mathcal{F}_{\mathrm{c} k}^{-1} E_{p}^{T} r_{p}(t)\right)$

\section{Cost Weighting Choice}

One of the main problems in nonlinear control is to ensure a stabilising control law and as the assumption at the start of the main Theorem 4.1 confirms this depends on the selection of the cost weightings. A practical method of deriving such weightings is suggested by these limiting results. The term $\left(I-\mathcal{F}_{\mathrm{c} k}^{-1} E_{p}^{T} P_{c} W_{0 k} W_{1 \mathrm{k}}\right)$ may be interpreted as the return-difference operator for a nonlinear system with delay-free plant modet $\mathcal{W}_{k}=W_{0 k} \mathcal{W}_{l k}$. Thus, if the plant already has a PID controller that stabilises this model, the ratio of weightings $\left(-\mathcal{F}_{c k}^{-1} E_{p}^{T} P_{c}\right)$ can be chosen equal to the $P I D$ controller. Then the weightings are linear and satisfy $F_{\mathrm{c} k}^{-1} E_{p}^{T} P_{c}=K_{P I D}$.

Even for a linear GPC design stability is not guaranteed for all cost function weightings. There will be some system descriptions where even scalar non-dynamic weightings may or not ensure a stable closed-loop design. Now if the cost horizon is reduced to one step the controller becomes equal to an $N G M V$ design, which can be guaranteed to have a stabilizing solution by a similar but simpler method as in the previous paragraph. The argument is then that as the multi-step cost is introduced and the horizon increases it is normally the case that predictive controls improve responses. Thus the strategy of starting with a well tuned $N G M V$ solution and then increasing the cost horizon to introduce predictive action is a practical method of finding cost terms that stabilize the closed loop. 


\section{Robustness of the Closed Loop System}

In the predictive control of linear systems it is usually the case that step response overshoots reduce as the cost horizon increases. This behaviour is related to smaller overshoots in frequency domain terms in the sensitivity functions and this suggests there is a commensurate improvement in robustness. Similar results are observed in the nonlinear case but robustness and sensitivity then relates to the sensitivity operators. The subject of robustness in the presence of plant uncertainties does of course deserve much more attention. A possible approach to the analysis of NPGMV systems is indicated briefly below.

Consider the case of a stable open loop system and note that the relationship (77) can be employed to show that the system in Fig. 4 can be redrawn as in Fig. 5 below. This figure is of interest because it resembles a Smith predictor type of structure but for the present discussion it allows robustness to be assessed in a particular case. Neglect for the moment most external inputs and assume that the plant has an additive uncertainty of the form: $\mathcal{W}=\overline{\mathcal{W}}+\Delta \mathcal{W}$. Then noting the signs of the signals summed in the bottom path observe that the diagram in Fig. 5 may be redrawn as shown in Fig. 6. From this diagram it is clear that the internal feedback loop which includes the delay free plant model has a significant effect on robustness. This loop depends on both the error and the control signal weighting choices. If for example the system has large high frequency uncertainty, such as resonances in a mechanical system, then the control costing can include a lead term introduced at frequencies below the possible resonant behaviour. The result will be a low gain in the forward path at high frequencies as determined by the inverse of the control costing within the inner loop. To some extent this weighting acts as a natural so called robustification filter. Zames (1966, [28]) small gain theorem can then be applied to demonstrate that an improvement in robustness has been achieved. 


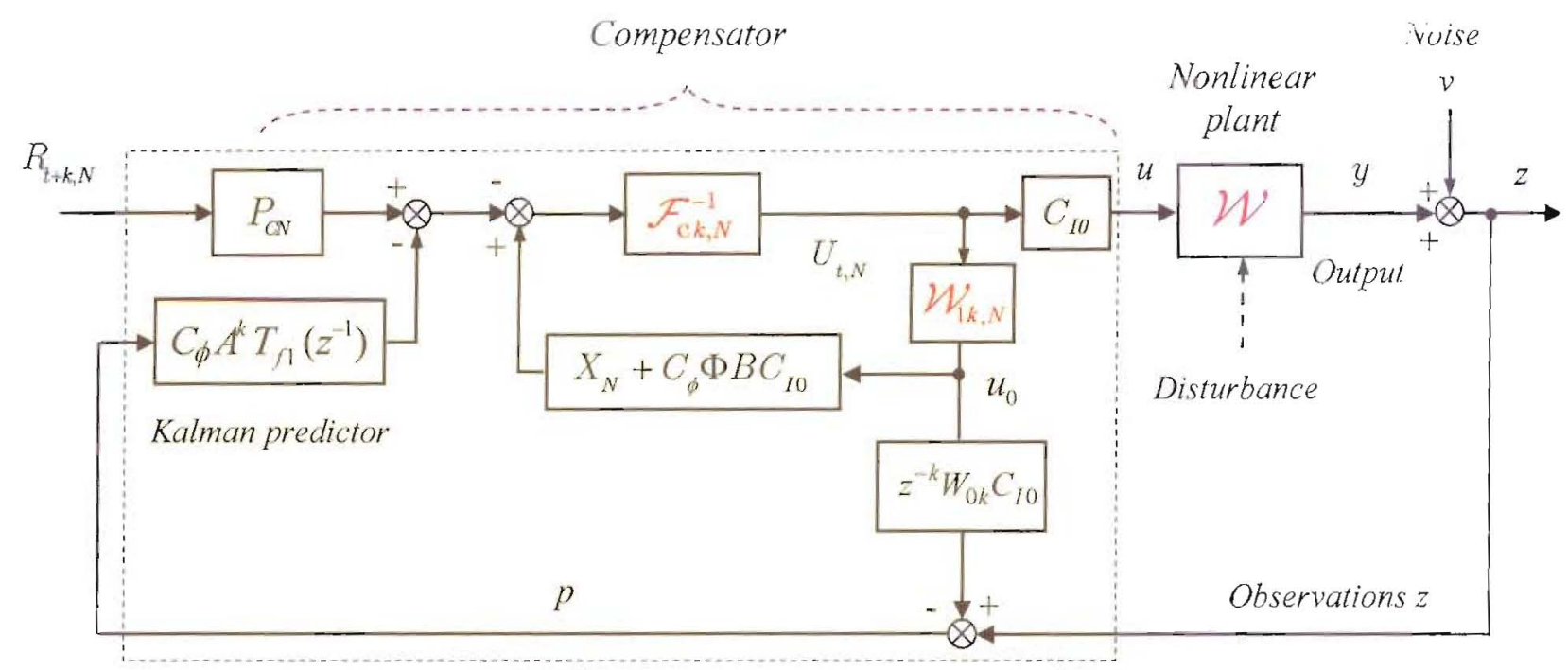

Fig. 5: Nonlinear Smith Predictor Implied by NPGMV Compensator Structure



Fig. 6: Loop Structure When Additive Uncertainty Present

\section{Predictive Control Design for Dynamic Ship Positioning}

One of the potential application areas for NPGMV control is in dynamic ship positioning and manoeuvring. A desired ship trajectory is often known in advance (in the case of dynamic ship positioning, the control objective normally consists in keeping the vessel's position constant, irrespective of wave/wind/current disturbances). Consider for example a ship given in the inertial 
Earth-based coordinate frame, as shown in Fig. 7. The objective here is to control the vector of ships position and heading $\eta=[x, y, \psi]^{\prime}$ via a thrusters/propeller propulsion system, so that a desired trajectory $\eta_{r e f}$ is followed. This is a well known problem that has been analyzed in detail in literature - see for example [14 to 17]. In the following, the NPGMV controller is used and assessed for this application.

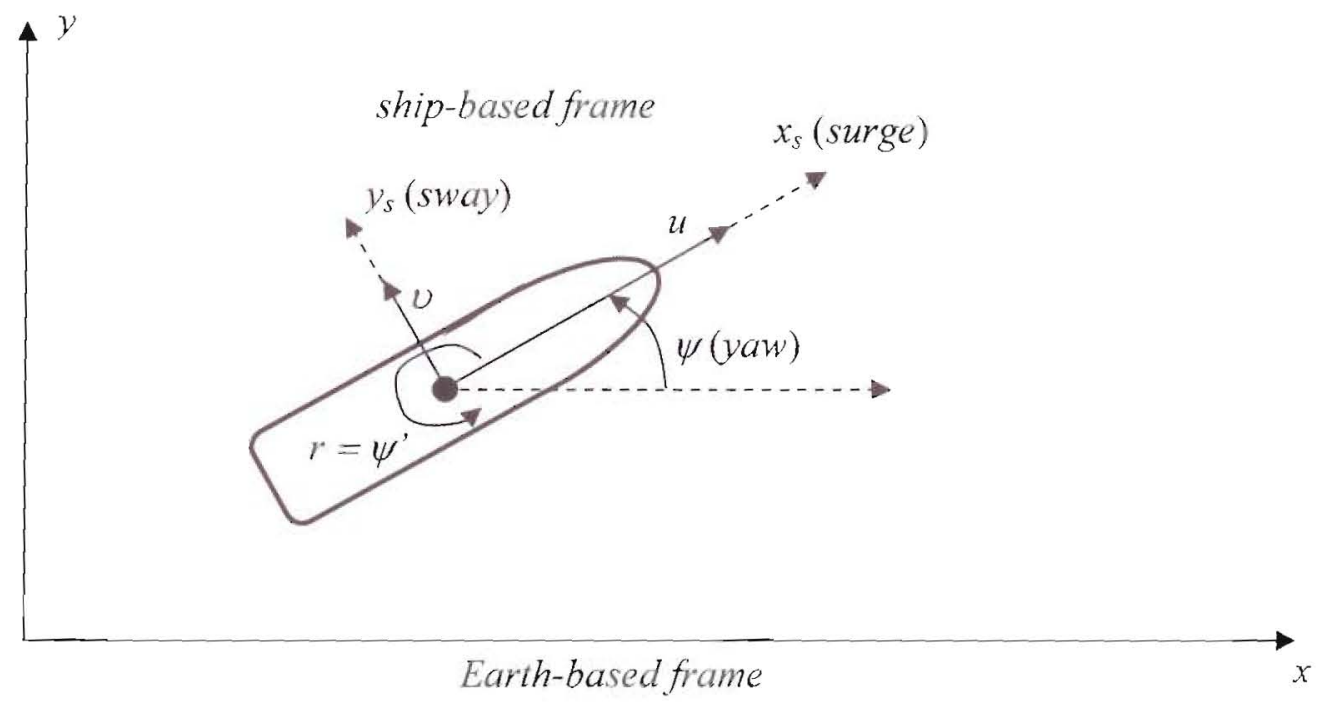

Fig. 7: Dynamic Ship Positioning Problem

System model: The simplified linearized dynamics of the system are described by the following differential equation:

$$
M \dot{v}+D v=\tau
$$

where $M$ is the inertia matrix, $D$ represents system damping, $v=\left[\begin{array}{lll}u & v & r\end{array}\right]^{T}$ is a ship-based velocity vector, and $\tau$ is a vector of forces in $x_{s}$ and $y_{s}$ directions, and the yaw torque. This approximation is valid especially at low speeds and positioning problems. The full nonlinear model, taking into account Coriolis and nonlinear damping terms, is given in [14].

The velocity vector is related to the Earth-based positions by the following kinematic equation:

$$
\dot{\eta}=R(\psi) v
$$


where $R(\psi)$ is the 3DOF rotation matrix: $R(\psi)=\left[\begin{array}{ccc}\cos \psi & -\sin \psi & 0 \\ \sin \psi & \cos \psi & 0 \\ 0 & 0 & 1\end{array}\right]$

A simple diagonal thruster configuration was assumed in this paper, and the following nonlinear static model for thrust forces and torque was used [15]:

$$
T_{1}=\rho d^{4} K_{l}\left(n_{i}\right)\left|n_{i}\right| n_{i} \quad i=1,2,3
$$

where $\rho$ is the water density, $d$ is the thruster diameter, $n$ is the velocity in $[\mathrm{rev} / \mathrm{s}]$, and $K_{T}$ is the additional nonlinear thruster coefficient. The nonlinear thruster characteristic as a function of $n$ is shown in Fig. 8. In the following simulation studies, we have used models from the Marine Systems Simulator Toolbox for Matlab [18].

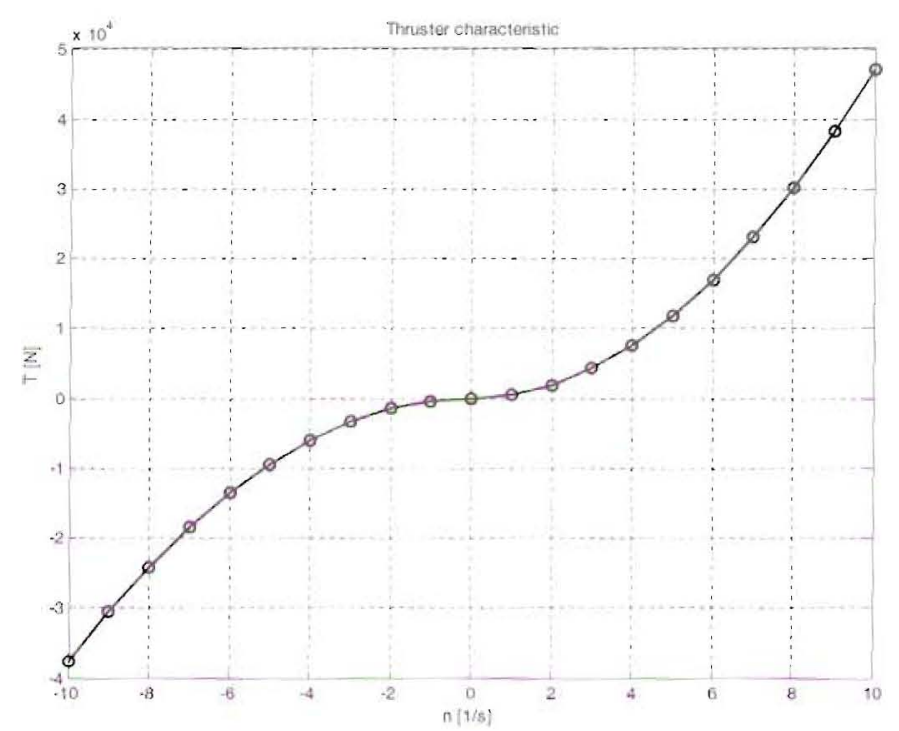

Fig. 8: Nonlinear Thrusters Characteristic

Based on the above system description, the open-loop Hammerstein model may be separated into nonlinear and linear components as shown in Fig. 9 , with the black-box model $W_{1 k}$ representing the thrusters. Note that the nonlinear transformation matrix $R(\psi)$ is not considered part of the subsystem $W_{0}$ and will only be used to plot the ship position in the inertial frame. 
For the controller design, a discrete model of the linear dynamics was obtained using the Tustin method, with the sample time $T_{s}=0.1 \mathrm{sec}$. A nominal one sample delay $(k=1)$ was assumed for this model.

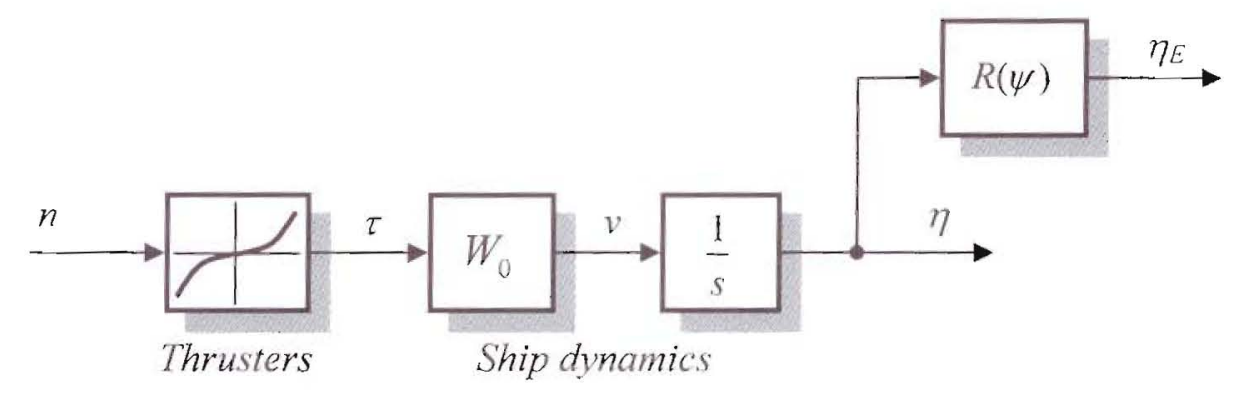

Fig. 9: Decomposition of the Ship Model

Wave model: The wave disturbance was described using a second order resonant system, according to the transfer function: $w(s)=\frac{k}{s^{2}+2 \zeta \omega_{n} s+\omega_{n}^{2}}$, where the parameters were defined as: $\omega_{n}=0.8 \frac{\mathrm{rad}}{\mathrm{s}}$ and $\zeta=0.1$. The scaling factor $k$ was chosen so that realistic wave amplitudes were obtained. The frequency responses of both the ship dynamics (not including the thrusters model) and the wave disturbance are plotted in Fig. 10. The main objective of the controller is to reduce the effect of the wave motion. 




Fig. 10: Frequency Responses of System Models (plant, disturbance and nominal error weight)

NPGMV Controller implementation: The structure of the model allows the solution of the algebraic loop problem to be simplified. Recall the expression for the optimal control sequence $(76)$ :

$$
U_{t, N}=-\mathcal{F}_{\mathrm{c} k, N}^{-1}\left(\tilde{R}_{t+k, N}^{0}-\left(X_{N}+\mathrm{C}_{\phi} T_{0}\left(k, z^{-1}\right) B C_{I 0}\right) \mathcal{W}_{1 \mathrm{k}, \mathrm{N}} U_{t, N}\right)
$$

The nonlinear thrusters model represented by $W_{1 k}$ is static and diagonal, while the control weighting can be separated into a component affected by the current control (the direct through term) and a part dependent only on the past control values: $\mathcal{F}_{c k}(\tau)=\mathcal{F}_{0}(\tau)+\mathcal{F}_{1}(\tau)$, where $\mathcal{F}_{1}(\tau)$ contains a one-step time-delay (note that a similar decomposition would apply to a dynamic $\mathcal{W}_{1 k}$ model). Employing block matrix notation to indicate the block-diagonal structure of $\mathcal{F}_{c k, N}$, the expression for the vector of future controls can be rewritten as:

$$
\left(\mathcal{F}_{0, N}+\mathcal{F}_{1, N}\right) U_{t, N}=-\left(\tilde{R}_{t+k, N}^{0}-\left(X_{N}+\mathrm{C}_{\phi} T_{0}\left(k, z^{-1}\right) B C_{I \theta}\right) \mathcal{W}_{1 \mathrm{k}, N} U_{t, N}\right)
$$

Regrouping the terms, an explicit expression for $U_{L N}$ follows as: 


$$
U_{t, N}=\left(\mathcal{F}_{0, N}-X_{N} \mathcal{W}_{1 \mathrm{k}, N}\right)^{-1}\left(-\tilde{R}_{t+k, N}^{0}-\left(\mathcal{F}_{1, N}-\mathrm{C}_{\phi} T_{0} B C_{I 0} \mathcal{W}_{1 \mathrm{k}, N}\right) U_{t, N}\right)
$$

Since $T_{0}\left(k, z^{-1}\right)$ contains a one-step time delay, the right-hand side of (88) involves the vector of controls computed in the previous step, and hence the current control is dependent on the past controls and inputs and the algebraic loop is removed.

For a practical realization of the control law, it is necessary to invert the static operator $\left(\mathcal{F}_{0, N}-X_{N} \mathcal{W}_{\mathrm{lk}, N}\right)$. Since $X_{N}$ is in general a full matrix, a closed analytical form of the inverse will not normally exist, even though the $\mathcal{W}_{1 k, N}$ nonlinearity is itself invertible. The solution is to solve the resulting nonlinear equation on-line, using an iterative Newton method [19]. An alternative is to insert a memory block inside the feedback loop - this leads to a realizable but suboptimal solution.

The final structure of the controller is as shown in Fig. 11. Note that the actuator saturation limits have also been included in the model.

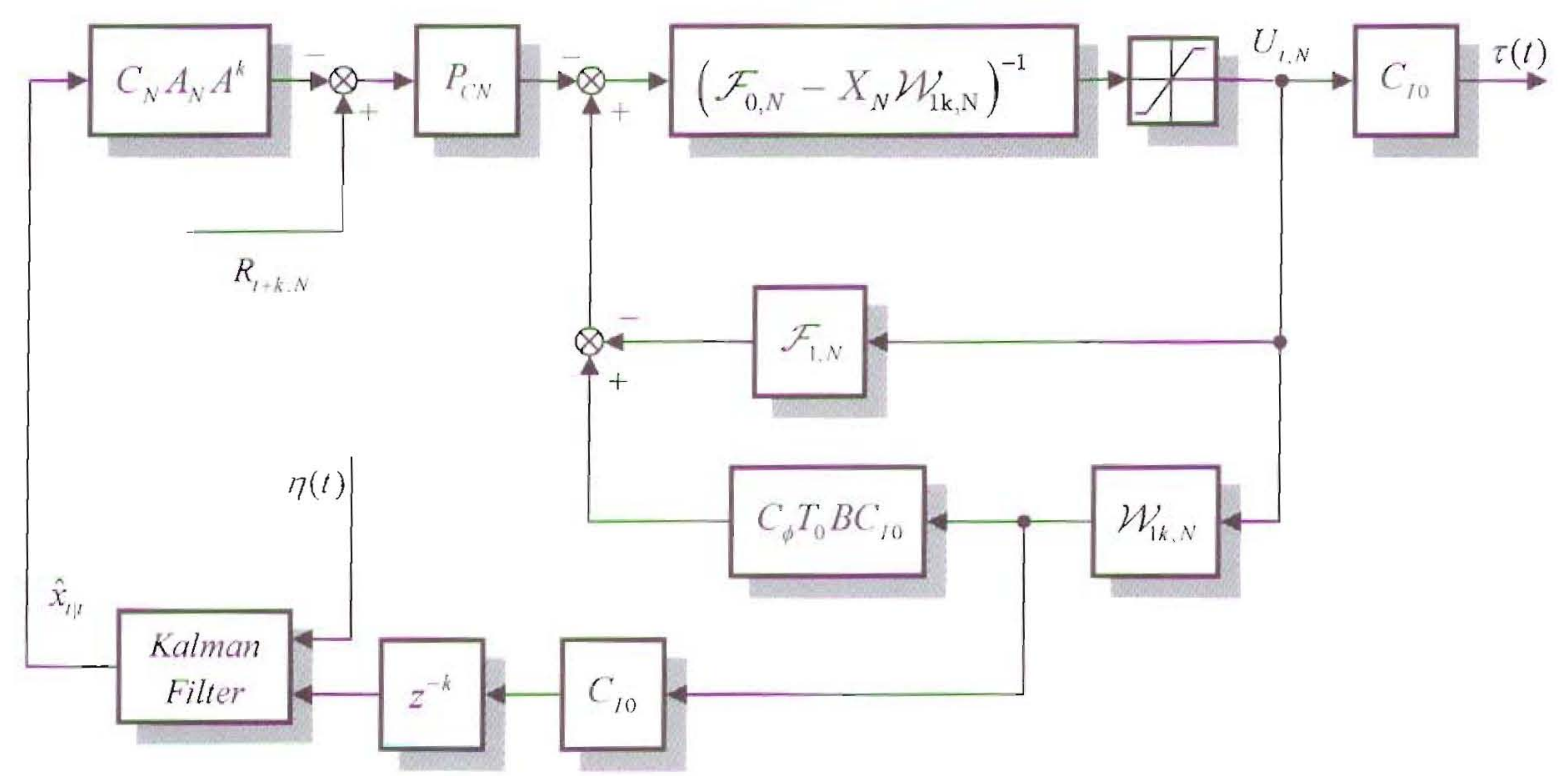

Fig. 11: Implementation of the NPGMV Controller 
Nominal Design: Since the system includes integrators (the controlled variables are assumed to be the position and heading), the controller does not normally require integral action - the PD structure is sufficient for perfect steady-state tracking. Following the tuning guidelines given in $\S 5.2$, the NPGMV controller design was initially based on such a $P D$ controller $C_{P D}\left(z^{-1}\right)$ with the tuning gains selected as $K_{p}=2 \times I$ and $K_{d}=\operatorname{diag}\{6,2,4\}$ (the derivative filters with time-constant $1 s$ were also included with the $D$ terms). The dynamic weightings were thus defined as $P_{c}\left(z^{-1}\right)=C_{P D}\left(z^{-1}\right)$ and $\mathcal{F}_{c k}=-V_{N}$, with the input weighting $\Lambda_{N}^{2}$ set to zero. As noted in Theorem 4.1, for the case of $N=0$, these weightings correspond to the limiting case of the $N G M V$ controller, whilst the horizon $N>0$ corresponds to the predictive control case.

Two simulation scenarios were considered: point positioning subject to wave motion and also elliptical trajectory following. The nominal simulation results for the limiting case are shown in Fig. 12 and 13. With the nominal weightings, the NPGMV control performance is close to that of $P D$ control (not shown) - this selection of weightings is thus a useful starting point for the design. The responses were intentionally made rather oscillatory in order to show the rapid improvement with increasing $N$ - the results that follow indicate that only a few steps are sufficient for a satisfactory design. 

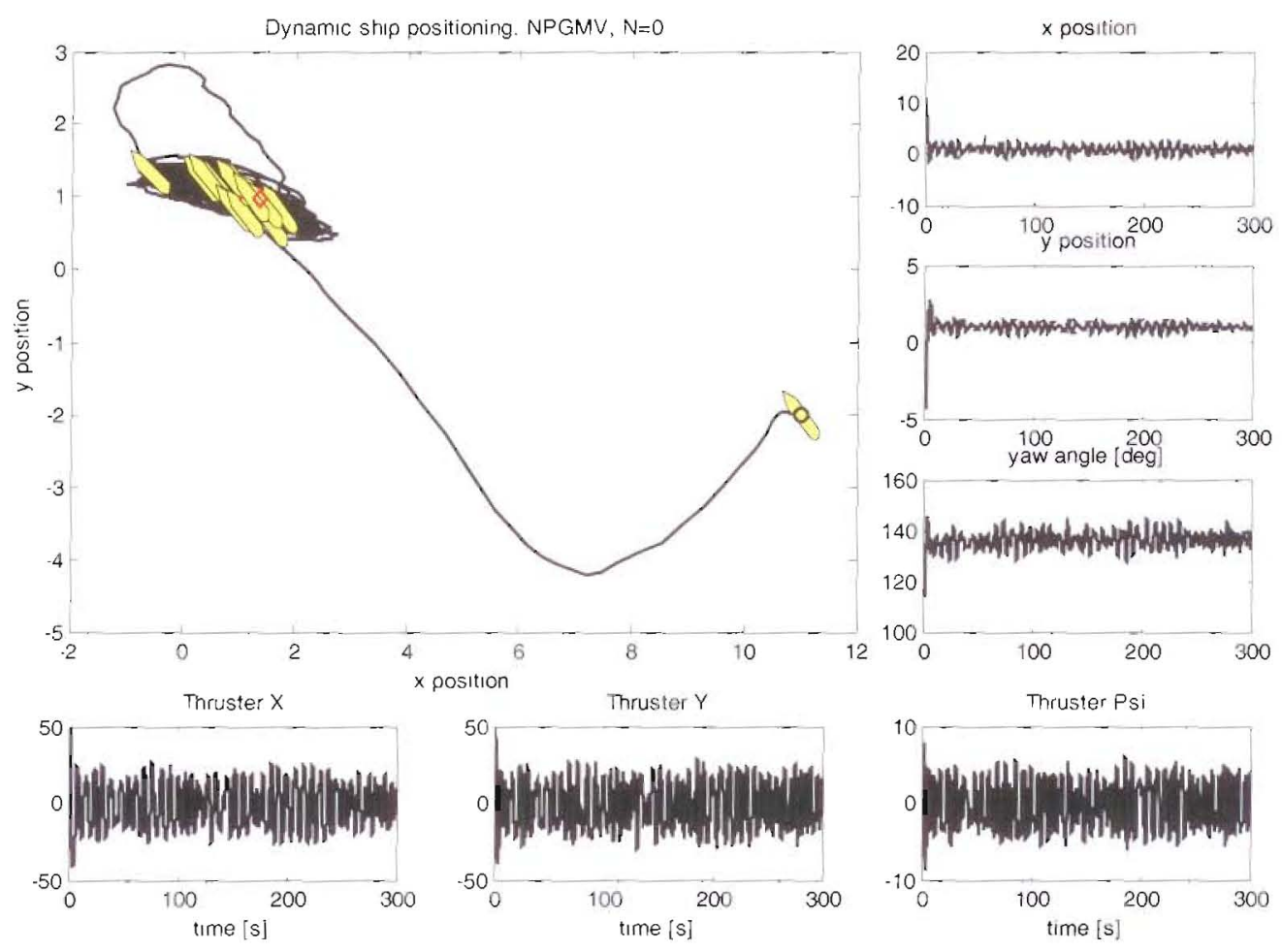
120
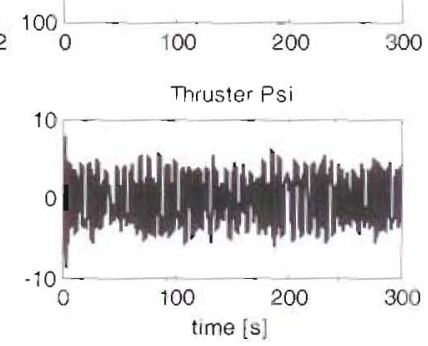

Fig. 12: Ship Positioning - NPGMV control with $N=0$
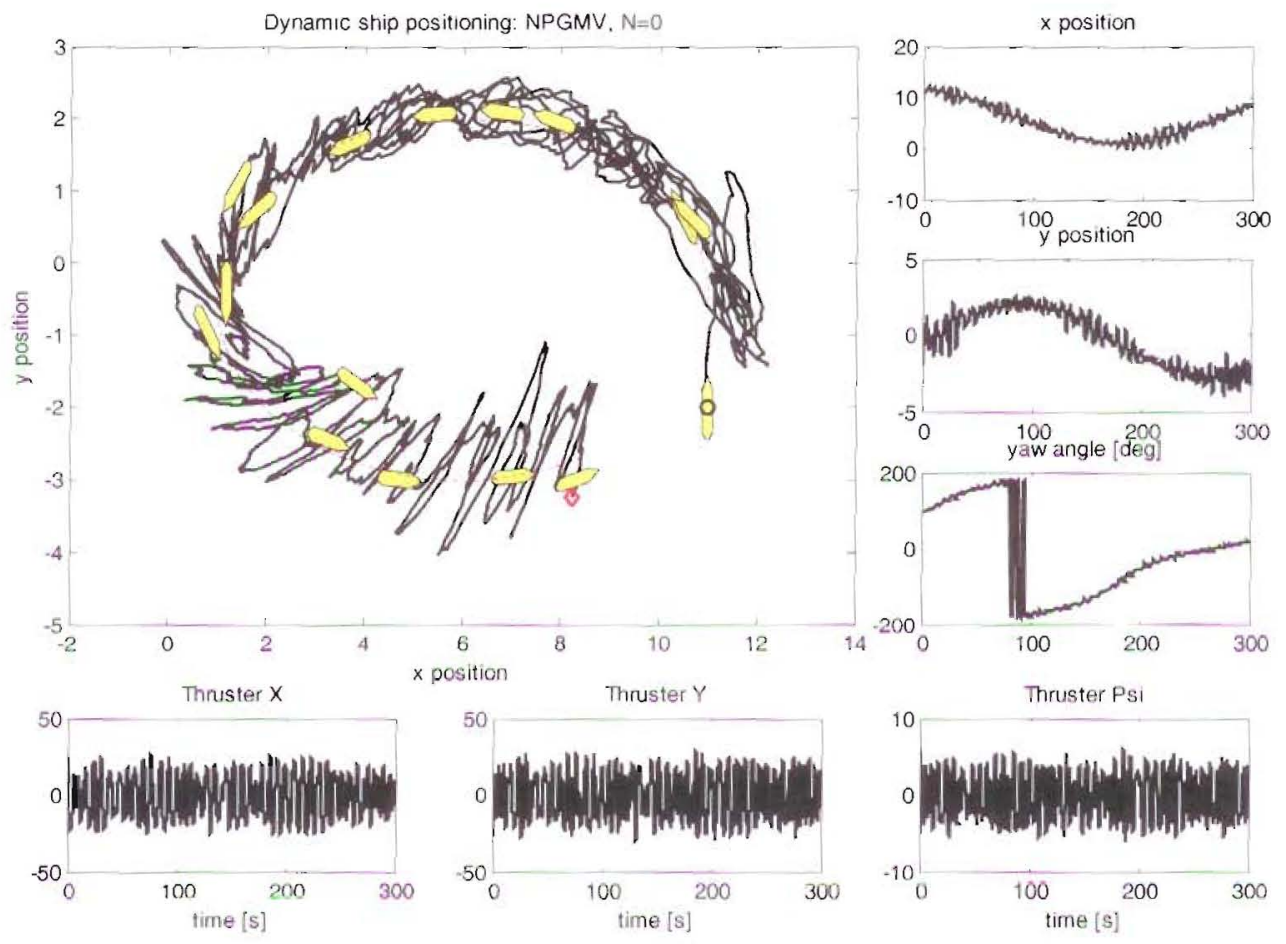

Fig. 13: Elliptic trajectory following - NPGMV control with $N=0$ 
Tuning Trials and Predictive Control: In the following modified design a lead term was included in the control weighting to reduce high-frequency noise amplification, while the error weighting $P_{c}\left(z^{-1}\right)$ was modified by penalizing the frequency band corresponding to the wave motion spectrum. This creates an inverted notch (bandpass) filter. In addition, the thrusters nonlinearities were used to redefine the control weighting as $\mathcal{F}_{c k}(u)=F_{c k}^{0} H(u)$ where $F_{c k}^{0}\left(z^{-1}\right)$ is the nominal linear control weighting, and $H(u)$ is the thrusters characteristic shown in Fig. 8. The inverse of $H$ was approximated by the expression: $n=4.6078 \cdot \operatorname{sig} n(T) \cdot \sqrt{|T|}$. Such definition takes into account the varying nonlinear gain of the actuators.

Simulation trials were performed for increasing values of the prediction horizon $N$. The responses for $N=3$ are shown in Figures $14-15$, and the reduced effect of the wave disturbance is evident when compared with Figs. 12-13. This is achieved at the expense of the more aggressive thrusters action (responding and compensating for the wave motion), however with saturation limits satisfied. 

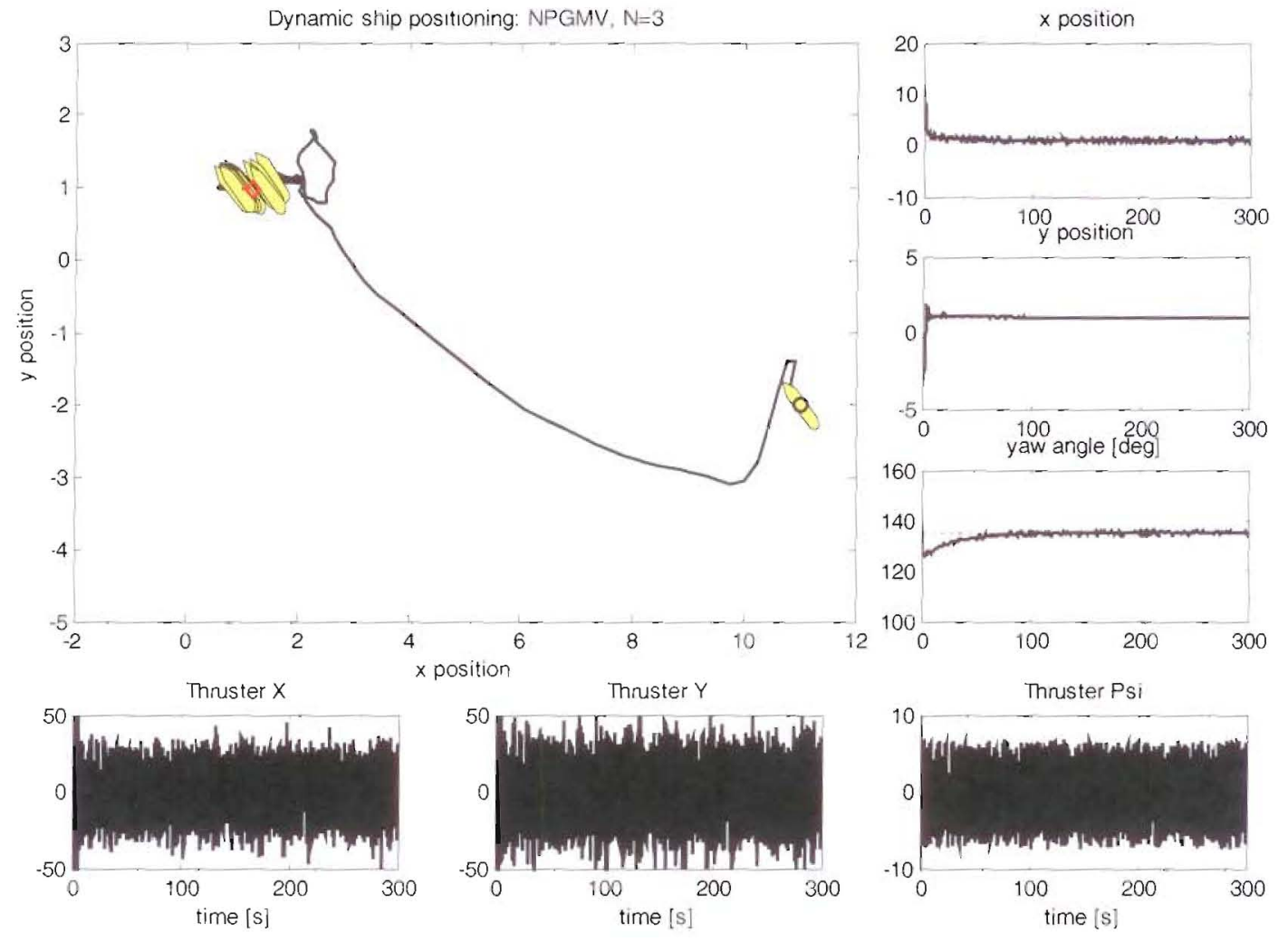

Fig. 14: Ship Positioning - Modified NPGMV control with $N=\mathbf{3}$ 

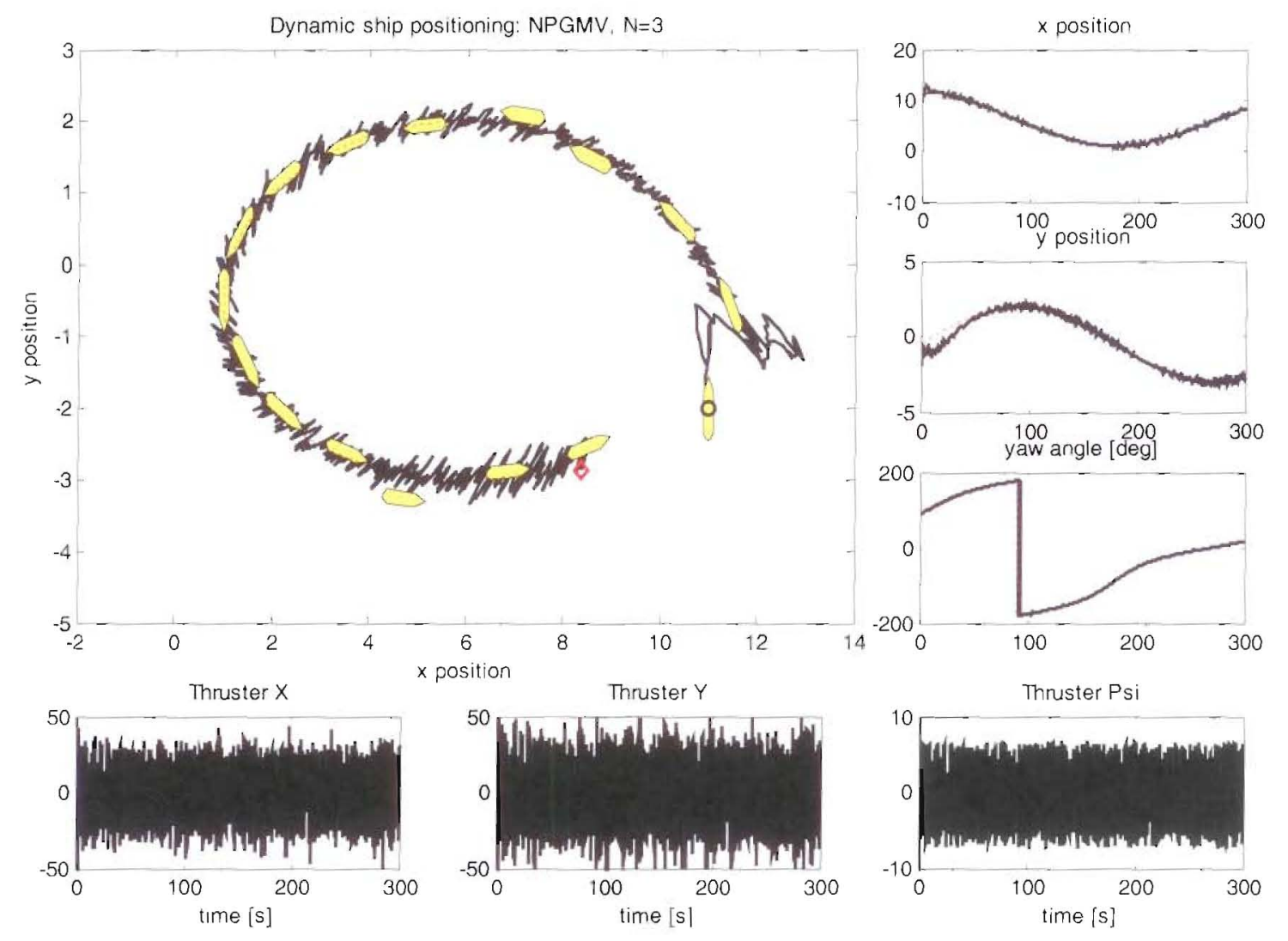

Fig. 15: Elliptic trajectory following - Modified NPGMV control with $N=3$

Final Remarks on the Example: Predictive control, when an extended prediction horizon is used, has the advantage that the control action can begin well before the changes in the reference signal occur, thanks to the future set point knowledge. This behavior is particularly useful in trajectory tracking applications and was demonstrated for the maneuvering subclass of the DP problem.

In addition to the nonlinear thruster characteristics in the controller internal model, the use of a nonlinear control weighting allowed to compensate for nonlinearities, while defining the dynamic weightings to penalize specific frequency ranges led to more effective wave disturbance rejection. The tuning procedure was facilitated by using the existing PD controller as a starting point for the design. The method does of course have its limitations; namely, the nonlinear part of the model is assumed to be stable, and the predictions are performed based on the linear subsystem, as in Fig. 
11. Despite these limitations, it may be concluded that the NPGMV offers some advantages relative to the basic $N G M V$ design, exploiting the well-known GPC control properties, at the expense of some additional complexity in the implementation. A generalization of this work will involve state-dependent models and will make use of the full nonlinear model of the ship.

\section{Concluding Remarks}

There are many nonlinear predictive control strategies based on ideas such as state-dependent models, linearization around a trajectory and others ([20] to [27]). However, the aim of the current development was to try to produce a control law which is related closely to fixed model based control and simple to implement. The NPGMV control design problem for a state-space system involved a multi-step predictive control cost-function and provided a method of introducing future set-point information. The predictive controls strategy described is a development of the $N G M V$ design method. It has the very nice property that if the system is linear then the control reverts to the Generalised Predictive Control design method which is well known and accepted in industry.

\section{References}

1. Cutler C.R. and Ramaker B.L., 1979, Dynamic matrix control - A computer control algorithm, A.I.C.H.E, 86th National Meeting, April 1979.

2. Clarke, D.W., C. Montadi and P.S. Tuffs, 1987, Generalized predictive control-Part I, The basic algorithm, Part 2, Extensions and interpretations, Automatica, 23, 2, pp.137-148.

3. Clarke, D.W., and C. Montadi, 1989, Properties of generalised predictive control, Automatica, Vol. 25, No. 6, pp. 859-875. 
4. Richalet J., A. Rault, J.L. Testud, J. Papon 1978, Model predictive heuristic control applications to industrial processes, Automatica, 14, pp. 413-428.

5. Richalet, J., 1993, Industrial applications of model based predictive control, Automatica, Vol. 29, No. 8, pp. 1251-1274.

6. Bitmead R., M. Gevers, and V. Wertz, 1990, Adaptive Optimal Control: The Thinking Man's GPC. Prentice Hall.

7. Ordys, A.W., and D.W. Clarke, 1993, A state-space description for GPC controllers, Int. J. Systems Science, Vol. 23, No. 2.

8. Grimble, M J, 2004, GMV control of nonlinear multivariable systems, UKACC Conference Control 2004, University of Bath, 6-9 September.

9. Grimble, M J, 2005, Non-linear generalised minimum variance feedback, feedforward and tracking control, Automatica, Vol. 41, pp 957-969.

10. Grimble, M J, and P Majecki, 2005, Nonlinear Generalised Minimum Variance Control Under Actuator Saturation, IFAC World Congress, Prague, Friday 8 July, 2005.

11. Grimble, M J, 2006, Robust industrial control, John Wiley, Chichester.

12. Grimble, M J, 2001, Industrial control systems design, John Wiley, Chichester.

13. Kwon W.H. and Pearson, A.E., 1977, A modified quadratic cost problem and feedback stabilization of a linear system, IEEE Transactions on Automatic Control, Vol. AC-22, No. 5 , pp. $838-842$.

14. Skjetne R, "The Maneuvering Problem", PhD thesis, 2005, Faculty of Information Technology, Mathematics, and Electrical Engineering Dept. of Eng. Cybernetics 
15 Fossen, T.I., Sagatun S.I. and A.J. Sorensen, "Identification of dynamically positioned ships", Control Eng. Practice, Vol. 4, No. 3, pp. 369-376, 1996

16 Katebi, M.R. Grimble M.J. and Y. Zhang, „Hळo robust control design for dynamic ship positioning", IEE Proceedings - Control Theory Appl., Vol. 144, No. 2, 1997

17 Fossen T. I., "Guidance and Control of Ocean Vehicles", John Wiley and Sons Ltd., 1994

18 Fossen, T. I. and T. Perez, 2004, Marine Systems Simulator (MSS), $<$ www.marinecontrol.org $>$.

19. Press W H., Teukolsky S A., Vetterling W T., and B. P. Flannery, 2002, Numerical Recipes in $\mathrm{C}++$ : The Art of Scientific Computing, Cambridge University press.

20. Michalska, H. and D.Q. Mayne, 1993, Robust receding horizon control of constrained nonlinear systems, IEEE Transactions on Automatic Control, 38, pp.1623-1633.

21. Kouvaritakis, B., M. Cannon and J.A. Rossiter, 1999, Nonlinear model based predictive control, Int. J. Control, 72(10), pp. 919-928.

22. Lee, Y.I., B. Kouvaritakis and M. Cannon, 2003, Constrained receding horizon predictive control for nonlinear systems, Automatica 38(12), pp. 2093-2102.

23. Mayne, D.Q., J.B. Rawlings, C.V. Rao and P.O.M. Scokaert, 2000, Constrained model predictive control: stability and optimality, Automatica 36(6), pp. 789-814.

24. Scokaert, P.O.M., D.Q. Mayne and J.B. Rawlings, 1999, Suboptimal model predictive control (feasibility implies stability), IEEE Transactions. Automatic Control, 44(3), pp. 648-654. 
25. Brooms, A.C. and B. Kouvaritakis, 2000, Successive constrained optimisation and interpolation in non-linear model based predictive control, Int. J. Control, 73(4), pp. 312316.

26. Allgower, F., and R. Findeisen, 1998, Non-linear predictive control of a distillation column, International Symposium on Non-linear Model Predictive Control, Ascona, Switzerland.

27. Camacho, E.F., 1993, Constrained generalized predictive control, IEEE Transactions on Automatic Control, 38, pp. 327-332.

28. Zames, G., 1966, On the input-output stability of time-varying nonlinear feedback systems. part i: Conditions derived using concepts of loop gain, conicity, and passivity," IEEE Trans. on Automatic Control, Vol. AC-11, no. 2, pp. 228-238. 